\title{
Modeling of Optimal Power Generation using Multiple Kites
}

\author{
Paul Williams, ${ }^{*}$ Bas Lansdorp, ${ }^{\dagger}$ Wubbo Ockels ${ }^{\ddagger}$ \\ Delft University of Technology, The Netherlands
}

\begin{abstract}
Kite systems have the potential to revolutionize energy generation. Large scale systems are envisioned that can fly autonomously in "power generation" cycles which drive a ground-based generator. In order for such systems to produce power efficiently, good models of the system are required. This paper focuses on the development of optimal open-loop trajectories for kite systems. In large-scale power generation, multiple kites connected along the same line will be able to produce significantly higher power due to the increase in the line tension. The equations of motion for a multikite power generating system are derived that take into account the key system parameters. Two different configurations are modeled: all kites are connected to the same line, multiple kites are connected to branches in the cable. The model can be used to estimate the power generation capabilities of various systems through dynamic optimization studies.
\end{abstract}

\section{Introduction}

W IND energy is an important source of potential power that has not yet been fully exploited. Over the last several decades, many proposals have been put forward for devices to extract and utilize wind energy. The most popular design is based on the wind turbines placed relatively close to the ground. However, it has long been known that the Earth's surface creates a boundary-layer-like effect on the wind so that the wind speed generally increases with altitude. In fact, it is possible for significant wind to be present at higher altitudes with little to no wind on the ground. This knowledge has spawned several radical ideas for wind power extraction. However, many of the basic principles of transmitting wind energy to the ground have been used since the early days of kite-flying, when kites were used for towing boats and wagons, ${ }^{1}$ or for lifting objects such as weather instrumentation. ${ }^{2}$ In these applications, a kite is flown so as to produce a beneficial lifting or pulling force that is transmitted to an object at lower altitude via a tether. However, as pointed out by Carpenter, ${ }^{1,2}$ with the advent of manned aircraft, such devices are seldom used today. One of the major problems encountered in the early application of kites for towing vehicles was the lack of adequate feedback control to cope with changing and unsteady winds. Modern day control techniques are capable of circumventing some of these problems, which has seen renewed interest in kite research. This paper is motivated by the desire to design an efficient system for extracting energy from the wind at high altitudes. Kites are envisioned to provide the optimal combination of lift-to-weight, together with an ability to be maneuvered, that make them particularly appealing for wind energy extraction.

One of the most promising applications of kites is for providing a means of generating electricity. ${ }^{3-11}$ By controlling the forces on the kite, thereby influencing the cable tension, it is possible to generate net power by utilizing a drum that is capable of paying the cable in- and out. If the tether is let out at high tension, it is generating power. If the tether is then reeled back in, it is using power. The key idea is to control the kite in such a way that the tension during the reel-in phase is much lower than the tension during the pay-out phase. The larger the difference in tension during reel-in and reel-out, the greater the power that can be generated. At the most basic level, if control of the kite motion can be achieved remotely, then it is possible to manipulate the tension in the tether by changing the forces on the kite. For example, to increase tension, the kite angle of attack can be increased, thereby increasing the lift on the kite. Conversely, to decrease tension, the kite angle of attack is decreased. Much more complex motions can be envisioned. The forces on the kite are a function of the kite angle of attack, its roll angle, and the local relative air speed. The most important of these for increasing the total lift force is the air speed, since doubling it results in a four-fold increase in lift.

The lift generated by a kite is directly proportional to the projected kite wing area. Hence, it is clear that larger systems can produce more power when flown at the same relative wind speeds. However, simply increasing the area of the kite can lead to larger issues related to stability and flexibility of the kite itself. Furthermore, it larger kites require larger cable diameters, which increases drag and decreases efficiency. There are two alternatives: 1)

\footnotetext{
* Applied Researcher, AIAA Member, e-mail: tethered.systems@gmail.com.

$\dagger$ PhD researcher, Faculty of Aerospace Engineering, E-mail: b.lansdorp@tudelft.nl.

*ASSET Chairholder, Faculty of Aerospace Engineering, E-mail: w.j.ockels@tudelft.nl.
} 
fly multiple kites on the same line, 2) fly multiple kites on multiple lines connected to the same ground station. This produces a better overall system design since the different cable segments can be designed to withstand their local maximum tensions. The lower portion of the cable can be thicker, but the wind speed is slower closer to the ground, so there is clearly an optimal distribution of kites and cables. This paper considers the modeling of the two types of kite systems. The full equations of motion are given for the case of two-kite systems and numerical simulations of power production are given to support the models.

\section{Dynamic Modeling of Tethered Kite System}

The type main configurations of the tethered kite system are shown in Fig. 1. Fig. 1a shows a series of kites connected on the same line, whereas the second configuration shown in Fig. 1b shows multiple kites connected to different lines that converge to the same power generation line. The idea of splitting the line was originally presented Ref. 12 .

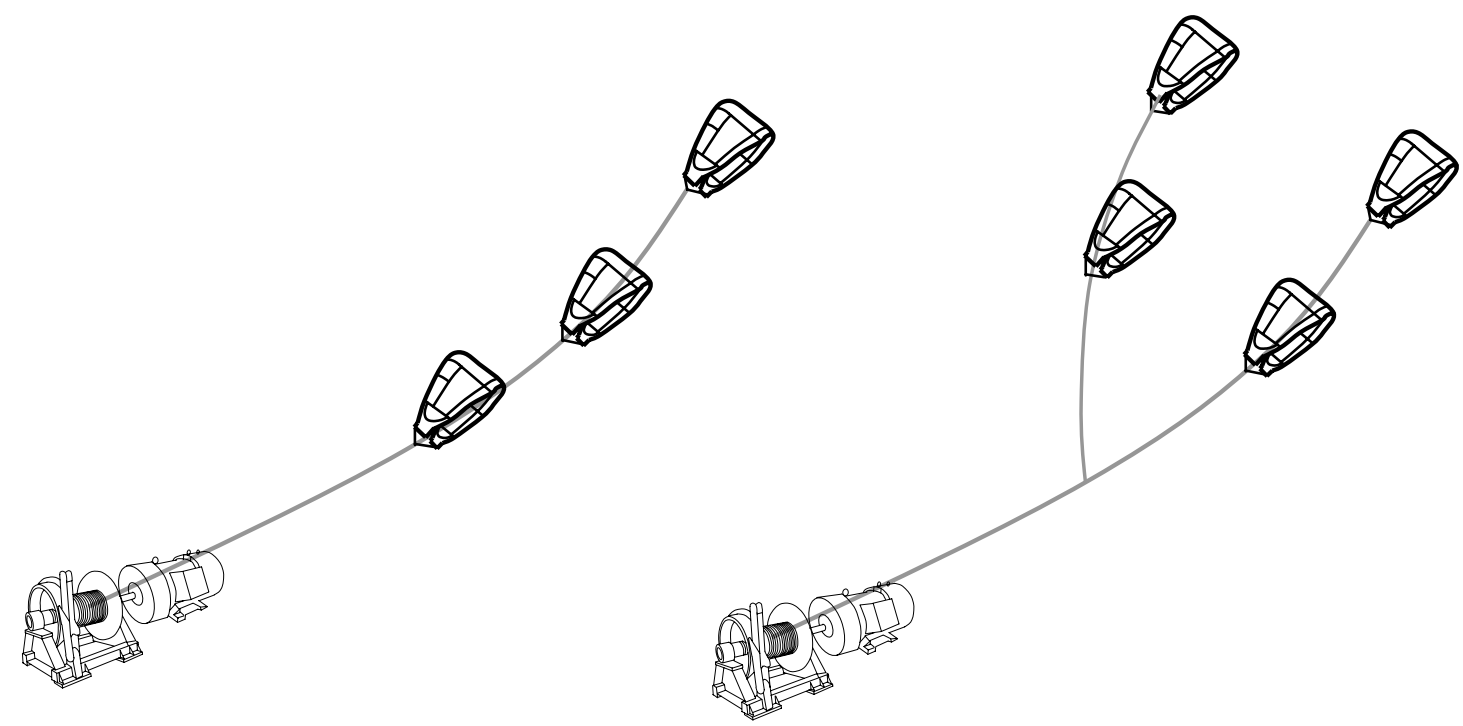

Fig. 1. Multiple kite power generation, a) Single line configuration, b) multiple line configuration.

\section{A. Case 1: Multiple Kites on Same Line}

The kite system is modeled in a simplified manner in order to enable optimal trajectory generation, as shown in Fig. 2. The tether(s) are modeled as inelastic and straight with a constant diameter. These assumptions were compared against more realistic lumped mass models in our previous work and found to produce representative results. ${ }^{3}$ The main reason for using such simplistic models of the tether is that it reduces the cable frequencies and enables rapid computation of system trajectories. However, in order for the tether model to reflect the dynamics of a higher fidelity model it is necessary to include the effect of tether mass and distributed drag along the cable. The equations of motion are derived using Lagrange's equations. 


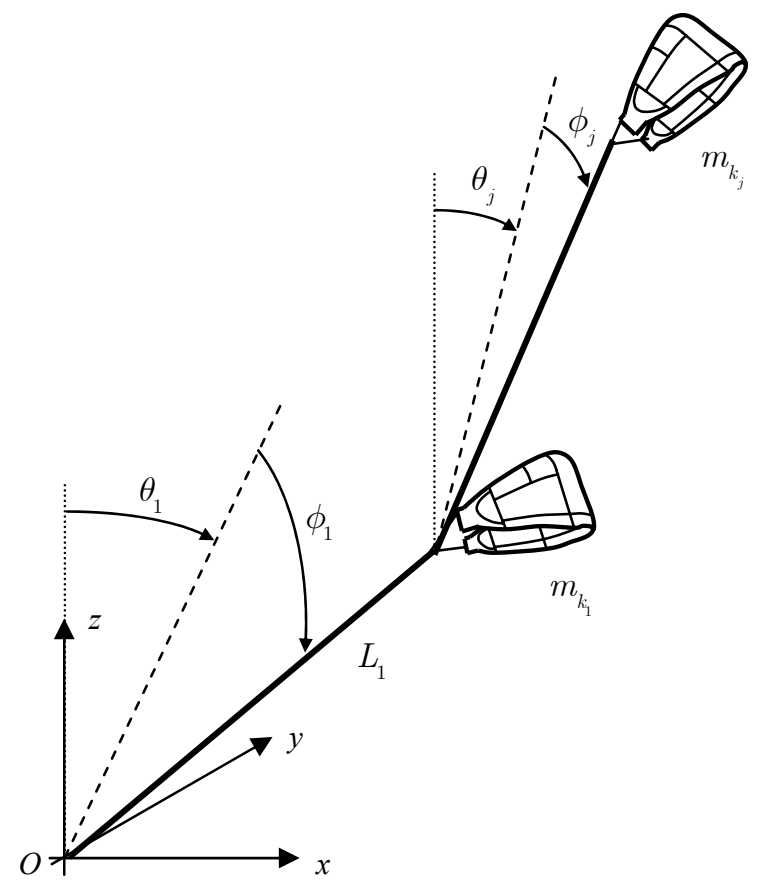

Fig. 2. Multiple kite model with kites on single line.

Consider the position of an element of tether mass $\rho_{c_{j}} \mathrm{~d} s_{j}$ on the $j$ th tether relative to the $(j-1)$ th kite. Consider its position in the inertial coordinate system. Using the spherical coordinates shown in Fig. 2 we have

$$
\boldsymbol{r}_{j}\left(s_{j}\right)=s_{j} \cos \phi_{j} \sin \theta_{j} \boldsymbol{i}+s_{j} \sin \phi_{j} \boldsymbol{j}+s_{j} \cos \phi_{j} \cos \theta_{j} \boldsymbol{k}
$$

The relative velocity is given by

$$
\begin{aligned}
\dot{\boldsymbol{r}}_{j}\left(s_{j}\right)= & \left(\dot{s}_{j} \cos \phi_{j} \sin \theta-s_{j} \dot{\phi}_{j} \sin \phi_{j} \sin \theta_{j}+s_{j} \dot{\theta}_{j} \cos \phi_{j} \cos \theta_{j}\right) \boldsymbol{i} \\
& +\left(\dot{s}_{j} \sin \phi_{j}+s_{j} \dot{\phi}_{j} \cos \phi_{j}\right) j \\
& +\left(\dot{s}_{j} \cos \phi_{j} \cos \theta_{j}-s_{j} \dot{\phi}_{j} \cos \phi_{j} \cos \theta_{j}-s_{j} \dot{\theta}_{j} \cos \phi_{j} \cos \theta_{j}\right) \boldsymbol{k}
\end{aligned}
$$

It should be noted that the tether(s) are assumed to be rigid such that $\dot{s}_{j}=\dot{L}_{j}$. The inertial position and total inertial velocity of the $j$ th element are given by

$$
\begin{aligned}
& \boldsymbol{R}_{j}\left(s_{j}\right)=\boldsymbol{r}_{j}\left(s_{j}\right)+\sum_{k=1}^{j-1} \boldsymbol{r}_{k}\left(L_{k}\right) \\
& \dot{\boldsymbol{R}}_{j}\left(s_{j}\right)=\dot{\boldsymbol{r}}_{j}\left(s_{j}\right)+\sum_{k=1}^{j-1} \dot{\boldsymbol{r}}_{k}\left(L_{k}\right)
\end{aligned}
$$

The kinetic energy of the system is given by the sum of the contributions from each tether and kite

$$
\mathcal{T}=\sum_{j=1}^{N} \frac{1}{2} \rho_{c_{j}} \int_{0}^{L_{j}} \dot{\boldsymbol{R}}_{j}\left(s_{j}\right) \cdot \dot{\boldsymbol{R}}_{j}\left(s_{j}\right) \mathrm{d} s_{j}+\left.\frac{1}{2} m_{k_{j}} \dot{\boldsymbol{R}}_{j}\left(s_{j}\right) \cdot \dot{\boldsymbol{R}}_{j}\left(s_{j}\right)\right|_{s_{j}=L_{j}}
$$

It is assumed that each tether has a uniform mass with line density $\rho_{c_{j}}$. The potential energy of the system is given by

$$
\mathcal{V}=\sum_{j=1}^{N} \rho_{c_{j}} g \int_{0}^{L_{j}} \boldsymbol{R}_{j}\left(s_{j}\right) \cdot \boldsymbol{k} \mathrm{d} s_{j}+\left.m_{k_{j}} g \boldsymbol{R}_{j}\left(s_{j}\right) \cdot \boldsymbol{k}\right|_{s_{j}=L_{j}}
$$


The kinetic energy may be evaluated as follows

$$
\begin{aligned}
\mathcal{T} & =\sum_{j=1}^{N} \frac{1}{2} \rho_{c_{j}} \int_{0}^{L_{j}}\left[\dot{\boldsymbol{r}}_{j}\left(s_{j}\right) \cdot \dot{\boldsymbol{r}}_{j}\left(s_{j}\right)+2 \dot{\boldsymbol{r}}_{j}\left(s_{j}\right) \cdot \sum_{k=1}^{j-1} \dot{\boldsymbol{r}}_{k}\left(L_{k}\right)+\left\{\sum_{k=1}^{j-1} \dot{\boldsymbol{r}}_{k}\left(L_{k}\right)\right\} \cdot\left\{\sum_{k=1}^{j-1} \dot{\boldsymbol{r}}_{k}\left(L_{k}\right)\right\}\right] \mathrm{d} s_{j} \\
& +\frac{1}{2} m_{k_{j}}\left[\left(\sum_{k=1}^{j} \dot{\boldsymbol{r}}_{k}\left(L_{k}\right)\right) \cdot\left(\sum_{k=1}^{j} \dot{\boldsymbol{r}}_{k}\left(L_{k}\right)\right]\right]
\end{aligned}
$$

where $m_{k_{j}}$ is the mass of the $j$ th kite.

\section{Two-Kite System}

We now proceed to explicitly derive the equations of motion for a two-kite system. Using Lagrange's equations the equations of motion can be expressed in the form

$$
[\boldsymbol{A}] \boldsymbol{q}=\boldsymbol{b}
$$

where $\boldsymbol{q}=\left[\ddot{\theta}_{1}, \ddot{\phi}_{1}, T_{1}, \ddot{\theta}_{2}, \ddot{\phi}_{2}, T_{2}\right]$ is a pseudo-acceleration vector that includes the unknown tether tensions. These must be solved simultaneously with the second derivatives of the generalized coordinates due to the assumption of rigid tethers. This assumption renders the second derivative of the tether lengths, $\ddot{L}_{j}$, as system control inputs. Note that knowledge of the tension is required for calculating the power output of the system.

The elements of the system matrix are

$$
\boldsymbol{A}=\left[\begin{array}{cccccc}
A_{1,1} & 0 & 0 & A_{1,4} & A_{1,5} & 0 \\
0 & A_{2,2} & 0 & A_{2,4} & A_{2,5} & 0 \\
0 & 0 & 1 & A_{3,4} & A_{3,5} & 0 \\
A_{1,4} & A_{2,4} & 0 & A_{4,4} & 0 & 0 \\
A_{1,5} & A_{2,5} & 0 & 0 & A_{5,5} & 0 \\
A_{6,1} & A_{6,2} & 0 & 0 & 0 & 1
\end{array}\right]
$$

where

$$
\begin{gathered}
A_{1,1}=\frac{1}{3} \rho_{1} L_{1}^{3} \cos ^{2} \phi_{1}+\rho_{2} L_{1}^{2} L_{2} \cos ^{2} \phi_{1}+\left(m_{1}+m_{2}\right) L_{1}^{2} \cos ^{2} \phi_{1} \\
A_{1,4}=\left(\frac{1}{2} \rho_{2} L_{1} L_{2}^{2}+m_{2} L_{1} L_{2}\right) \cos \phi_{2} \cos \phi_{1}\left(\sin \theta_{2} \sin \theta_{1}+\cos \theta_{2} \cos \theta_{1}\right) \\
A_{1,5}=\left(\frac{1}{2} \rho_{2} L_{1} L_{2}^{2}+m_{2} L_{1} L_{2}\right) \sin \phi_{2} \cos \phi_{1}\left(-\sin \theta_{2} \cos \theta_{1}+\cos \theta_{2} \sin \theta_{1}\right) \\
A_{2,2}=\frac{1}{3} \rho_{1} L_{1}^{3}+\rho_{2} L_{2} L_{1}^{2}+m_{1} L_{1}^{2}+m_{2} L_{1}^{2} \\
A_{2,5}=\left(\frac{1}{2} \rho_{2} L_{1} L_{2}^{2}+m_{2} L_{1} L_{2}\right)\left(\cos \phi_{2} \cos \phi_{1}+\sin \phi_{2} \sin \theta_{2} \sin \phi_{1} \sin \theta_{1}+\sin \phi_{2} \cos \theta_{2} \sin \phi_{1} \cos \theta_{1}\right) \\
A_{2,4}=\left(\frac{1}{2} \rho_{2} L_{1} L_{2}^{2}+m_{2} L_{1} L_{2}\right) \cos \phi_{2} \sin \phi_{1}\left(\sin \theta_{2} \cos \theta_{1}-\cos \theta_{2} \sin \theta_{1}\right) \\
A_{3,4}=\left(\frac{1}{2} \rho_{2} L_{2}^{2}+m_{2} L_{2}\right)\left(\sin \theta_{1} \cos \theta_{2}-\sin \theta_{2} \cos \theta_{1}\right) \cos \phi_{2} \cos \phi_{1} \\
A_{3,5}=\left(\frac{1}{2} \rho_{2} L_{2}^{2}+m_{2} L_{2}\right)\left(\cos \phi_{2} \sin \phi_{1}-\sin \phi_{2} \sin \theta_{2} \cos \phi_{1} \sin \theta_{1}-\sin \phi_{2} \cos \theta_{2} \cos \phi_{1} \cos \theta_{1}\right) \\
A_{4,4}=\left(m_{2}+\frac{1}{3} \rho_{2} L_{2}\right) L_{2}^{2} \cos \phi_{2} \\
A_{5,5}=\left(m_{2}+\frac{1}{3} \rho_{2} L_{2}\right) L_{2}^{2} \\
A_{6,2}=\left(\frac{1}{2} \rho_{2} L_{1} L_{2}+m_{2} L_{1}\right)\left(\cos \phi_{1} \sin \phi_{2}-\cos \phi_{2} \cos \theta_{2} \sin \phi_{1} \cos \theta_{1}-\cos \phi_{2} \sin \theta_{2} \sin \phi_{1} \sin \theta_{1}\right) \\
\left.A_{2} L_{1} L_{2}+m_{2} L_{1}\right) \cos \phi_{2} \cos \phi_{1}\left(\sin \theta_{2} \cos \theta_{1}-\cos \theta_{2} \sin \theta_{1}\right)
\end{gathered}
$$


It should be noted that the system matrix $A$ is not completely symmetric due to the fact that the inelastic constraints are being solved for rather than the second derivative of length with specified tensions. The components of the right-hand side of the equations of motion are given by

$$
\begin{aligned}
& b_{1}=\left[2 \rho_{2} L_{1}^{2} \cos \left(\phi_{1}\right) L_{2} \sin \left(\phi_{1}\right)+2 m_{1} L_{1}^{2} \cos \left(\phi_{1}\right) \sin \left(\phi_{1}\right)+2 m_{2} L_{1}^{2} \cos \left(\phi_{1}\right) \sin \left(\phi_{1}\right)\right. \\
& \left.+2 / 3 \rho_{1} \cos \left(\phi_{1}\right) L_{1}^{3} \sin \left(\phi_{1}\right)\right) \dot{\theta}_{1} \dot{\phi}_{1}+\left(-\rho_{1}\left(\cos \left(\phi_{1}\right)\right)^{2} L_{1}^{2} \dot{L}_{1}-2 m_{2} L_{1}\left(\cos \left(\phi_{1}\right)\right)^{2} \dot{L}_{1}\right. \\
& \left.-2 \rho_{2} L_{1}\left(\cos \left(\phi_{1}\right)\right)^{2} L_{2} \dot{L}_{1}-\rho_{2} L_{1}^{2}\left(\cos \left(\phi_{1}\right)\right)^{2} \dot{L}_{2}-2 m_{1} L_{1}\left(\cos \left(\phi_{1}\right)\right)^{2} \dot{L}_{1}\right) \dot{\theta}_{1} \\
& +\left(1 / 2 \rho_{2} L_{2}{ }^{2} \cos \left(\phi_{2}\right) \sin \left(\theta_{2}\right) L_{1} \cos \left(\phi_{1}\right) \cos \left(\theta_{1}\right)+m_{2} L_{2} \cos \left(\phi_{2}\right) \sin \left(\theta_{2}\right) L_{1} \cos \left(\phi_{1}\right) \cos \left(\theta_{1}\right)\right. \\
& \left.-m_{2} L_{2} \cos \left(\phi_{2}\right) \cos \left(\theta_{2}\right) L_{1} \cos \left(\phi_{1}\right) \sin \left(\theta_{1}\right)-1 / 2 \rho_{2} L_{2}{ }^{2} \cos \left(\phi_{2}\right) \cos \left(\theta_{2}\right) L_{1} \cos \left(\phi_{1}\right) \sin \left(\theta_{1}\right)\right) \dot{\theta}_{2}^{2} \\
& +\left(\left(\rho_{2} L_{2}^{2} \sin \left(\phi_{2}\right) \cos \left(\theta_{2}\right) L_{1} \cos \left(\phi_{1}\right) \cos \left(\theta_{1}\right)+2 m_{2} L_{2} \sin \left(\phi_{2}\right) \cos \left(\theta_{2}\right) L_{1} \cos \left(\phi_{1}\right) \cos \left(\theta_{1}\right)\right.\right. \\
& \left.+2 m_{2} L_{2} \sin \left(\phi_{2}\right) \sin \left(\theta_{2}\right) L_{1} \cos \left(\phi_{1}\right) \sin \left(\theta_{1}\right)+\rho_{2} L_{2}^{2} \sin \left(\phi_{2}\right) \sin \left(\theta_{2}\right) L_{1} \cos \left(\phi_{1}\right) \sin \left(\theta_{1}\right)\right) \dot{\phi}_{2} \\
& -2 \rho_{2} \dot{L}_{2} \cos \left(\phi_{2}\right) \sin \left(\theta_{2}\right) L_{1} \cos \left(\phi_{1}\right) \sin \left(\theta_{1}\right) L_{2}-2 m_{2} \dot{L}_{2} \cos \left(\phi_{2}\right) \cos \left(\theta_{2}\right) L_{1} \cos \left(\phi_{1}\right) \cos \left(\theta_{1}\right) \\
& \left.-2 m_{2} \dot{L}_{2} \cos \left(\phi_{2}\right) \sin \left(\theta_{2}\right) L_{1} \cos \left(\phi_{1}\right) \sin \left(\theta_{1}\right)-2 \rho_{2} \dot{L}_{2} \cos \left(\phi_{2}\right) \cos \left(\theta_{2}\right) L_{1} \cos \left(\phi_{1}\right) \cos \left(\theta_{1}\right) L_{2}\right) \dot{\theta}_{2} \\
& +\left(1 / 2 \rho_{2} L_{2}^{2} \cos \left(\phi_{2}\right) \sin \left(\theta_{2}\right) L_{1} \cos \left(\phi_{1}\right) \cos \left(\theta_{1}\right)+m_{2} L_{2} \cos \left(\phi_{2}\right) \sin \left(\theta_{2}\right) L_{1} \cos \left(\phi_{1}\right) \cos \left(\theta_{1}\right)\right. \\
& \left.-m_{2} L_{2} \cos \left(\phi_{2}\right) \cos \left(\theta_{2}\right) L_{1} \cos \left(\phi_{1}\right) \sin \left(\theta_{1}\right)-1 / 2 \rho_{2} L_{2}{ }^{2} \cos \left(\phi_{2}\right) \cos \left(\theta_{2}\right) L_{1} \cos \left(\phi_{1}\right) \sin \left(\theta_{1}\right)\right) \dot{\phi}_{2}{ }^{2} \\
& +\left(2 \rho_{2} \dot{L}_{2} \sin \left(\phi_{2}\right) \sin \left(\theta_{2}\right) L_{1} \cos \left(\phi_{1}\right) \cos \left(\theta_{1}\right) L_{2}-2 m_{2} \dot{L}_{2} \sin \left(\phi_{2}\right) \cos \left(\theta_{2}\right) L_{1} \cos \left(\phi_{1}\right) \sin \left(\theta_{1}\right)\right. \\
& \left.+2 m_{2} \dot{L}_{2} \sin \left(\phi_{2}\right) \sin \left(\theta_{2}\right) L_{1} \cos \left(\phi_{1}\right) \cos \left(\theta_{1}\right)-2 \rho_{2} \dot{L}_{2} \sin \left(\phi_{2}\right) \cos \left(\theta_{2}\right) L_{1} \cos \left(\phi_{1}\right) \sin \left(\theta_{1}\right) L_{2}\right) \dot{\phi}_{2} \\
& +\rho_{2} \dot{L}_{2}^{2} \cos \left(\phi_{2}\right) \cos \left(\theta_{2}\right) L_{1} \cos \left(\phi_{1}\right) \sin \left(\theta_{1}\right)+m_{2} L_{1} \cos \left(\phi_{1}\right) \sin \left(\theta_{1}\right)+\rho_{2} g L_{1} \cos \left(\phi_{1}\right) \sin \left(\theta_{1}\right) L_{2} \\
& +m_{1} L_{1} \cos \left(\phi_{1}\right) \sin \left(\theta_{1}\right)-\rho_{2} \ddot{L}_{2} \cos \left(\phi_{2}\right) \sin \left(\theta_{2}\right) L_{1} \cos \left(\phi_{1}\right) \cos \left(\theta_{1}\right) L_{2} \\
& +m_{2} \ddot{L}_{2} \cos \left(\phi_{2}\right) \cos \left(\theta_{2}\right) L_{1} \cos \left(\phi_{1}\right) \sin \left(\theta_{1}\right)-\rho_{2} \dot{L}_{2}^{2} \cos \left(\phi_{2}\right) \sin \left(\theta_{2}\right) L_{1} \cos \left(\phi_{1}\right) \cos \left(\theta_{1}\right) \\
& +1 / 2 \rho_{1} g \cos \left(\phi_{1}\right) \sin \left(\theta_{1}\right) L_{1}^{2}+\rho_{2} \ddot{L}_{2} \cos \left(\phi_{2}\right) \cos \left(\theta_{2}\right) L_{1} \cos \left(\phi_{1}\right) \sin \left(\theta_{1}\right) L_{2} \\
& -m_{2} \ddot{L}_{2} \cos \left(\phi_{2}\right) \sin \left(\theta_{2}\right) L_{1} \cos \left(\phi_{1}\right) \cos \left(\theta_{1}\right)+Q_{1}
\end{aligned}
$$

$$
\begin{aligned}
& b_{2}=\left(-\rho_{1} L_{1}^{2} \dot{L}_{1}-\rho_{2} L_{1}^{2} \dot{L}_{2}-2 m_{1} L_{1} \dot{L}_{1}-2 m_{2} L_{1} \dot{L}_{1}-2 \rho_{2} L_{1} L_{2} \dot{L}_{1}\right) \dot{\phi}_{1} \\
& +\left(-1 / 3 \rho_{1} \cos \left(\phi_{1}\right) L_{1}^{3} \sin \left(\phi_{1}\right)-m_{1} L_{1}^{2} \cos \left(\phi_{1}\right) \sin \left(\phi_{1}\right)-\rho_{2} L_{1}^{2} \cos \left(\phi_{1}\right) L_{2} \sin \left(\phi_{1}\right)-m_{2} L_{1}^{2} \cos \left(\phi_{1}\right) \sin \left(\phi_{1}\right)\right) \dot{\theta}_{1}^{2} \\
& +\left(-m_{2} L_{2} \cos \left(\phi_{2}\right) \sin \left(\theta_{2}\right) L_{1} \sin \left(\phi_{1}\right) \sin \left(\theta_{1}\right)-1 / 2 \rho_{2} L_{2}^{2} \cos \left(\phi_{2}\right) \cos \left(\theta_{2}\right) L_{1} \sin \left(\phi_{1}\right) \cos \left(\theta_{1}\right)\right. \\
& -1 / 2 \rho_{2} L_{2}^{2} \cos \left(\phi_{2}\right) \sin \left(\theta_{2}\right) L_{1} \sin \left(\phi_{1}\right) \sin \left(\theta_{1}\right)-m_{2} L_{2} \cos \left(\phi_{2}\right) \cos \left(\theta_{2}\right) L_{1} \sin \left(\phi_{1}\right) \cos \left(\theta_{1}\right) \dot{\theta}_{2}^{2} \\
& +\left(\left(-\rho_{2} L_{2}^{2} \sin \left(\phi_{2}\right) \cos \left(\theta_{2}\right) L_{1} \sin \left(\phi_{1}\right) \sin \left(\theta_{1}\right)+\rho_{2} L_{2}^{2} \sin \left(\phi_{2}\right) \sin \left(\theta_{2}\right) L_{1} \sin \left(\phi_{1}\right) \cos \left(\theta_{1}\right)\right.\right. \\
& \left.-2 m_{2} L_{2} \sin \left(\phi_{2}\right) \cos \left(\theta_{2}\right) L_{1} \sin \left(\phi_{1}\right) \sin \left(\theta_{1}\right)+2 m_{2} L_{2} \sin \left(\phi_{2}\right) \sin \left(\theta_{2}\right) L_{1} \sin \left(\phi_{1}\right) \cos \left(\theta_{1}\right)\right) \dot{\phi}_{2} \\
& -2 \rho_{2} \dot{L}_{2} \cos \left(\phi_{2}\right) \sin \left(\theta_{2}\right) L_{1} \sin \left(\phi_{1}\right) \cos \left(\theta_{1}\right) L_{2}+2 m_{2} \dot{L}_{2} \cos \left(\phi_{2}\right) \cos \left(\theta_{2}\right) L_{1} \sin \left(\phi_{1}\right) \sin \left(\theta_{1}\right) \\
& \left.+2 \rho_{2} \dot{L}_{2} \cos \left(\phi_{2}\right) \cos \left(\theta_{2}\right) L_{1} \sin \left(\phi_{1}\right) \sin \left(\theta_{1}\right) L_{2}-2 m_{2} \dot{L}_{2} \cos \left(\phi_{2}\right) \sin \left(\theta_{2}\right) L_{1} \sin \left(\phi_{1}\right) \cos \left(\theta_{1}\right)\right) \dot{\theta}_{2} \\
& +\left(-m_{2} L_{2} \cos \left(\phi_{2}\right) \sin \left(\theta_{2}\right) L_{1} \sin \left(\phi_{1}\right) \sin \left(\theta_{1}\right)+m_{2} L_{2} \sin \left(\phi_{2}\right) L_{1} \cos \left(\phi_{1}\right)+1 / 2 \rho_{2} L_{2}^{2} \sin \left(\phi_{2}\right) L_{1} \cos \left(\phi_{1}\right)\right. \\
& -1 / 2 \rho_{2} L_{2}^{2} \cos \left(\phi_{2}\right) \sin \left(\theta_{2}\right) L_{1} \sin \left(\phi_{1}\right) \sin \left(\theta_{1}\right)-1 / 2 \rho_{2} L_{2}^{2} \cos \left(\phi_{2}\right) \cos \left(\theta_{2}\right) L_{1} \sin \left(\phi_{1}\right) \cos \left(\theta_{1}\right) \\
& \left.-m_{2} L_{2} \cos \left(\phi_{2}\right) \cos \left(\theta_{2}\right) L_{1} \sin \left(\phi_{1}\right) \cos \left(\theta_{1}\right)\right) \dot{\phi}_{2}^{2}+\left(-2 \rho_{2} \dot{L}_{2} \cos \left(\phi_{2}\right) L_{1} \cos \left(\phi_{1}\right) L_{2}\right. \\
& -2 \rho_{2} \dot{L}_{2} \sin \left(\phi_{2}\right) \cos \left(\theta_{2}\right) L_{1} \sin \left(\phi_{1}\right) \cos \left(\theta_{1}\right) L_{2}-2 m_{2} \dot{L}_{2} \sin \left(\phi_{2}\right) \sin \left(\theta_{2}\right) L_{1} \sin \left(\phi_{1}\right) \sin \left(\theta_{1}\right) \\
& -2 \rho_{2} \dot{L}_{2} \sin \left(\phi_{2}\right) \sin \left(\theta_{2}\right) L_{1} \sin \left(\phi_{1}\right) \sin \left(\theta_{1}\right) L_{2}-2 m_{2} \dot{L}_{2} \sin \left(\phi_{2}\right) \cos \left(\theta_{2}\right) L_{1} \sin \left(\phi_{1}\right) \cos \left(\theta_{1}\right) \\
& \left.-2 m_{2} \dot{L}_{2} \cos \left(\phi_{2}\right) L_{1} \cos \left(\phi_{1}\right)\right) \dot{\phi}_{2}+m_{1} L_{1} \sin \left(\phi_{1}\right) \cos \left(\theta_{1}\right)+m_{2} L_{1} \sin \left(\phi_{1}\right) \cos \left(\theta_{1}\right)+\rho_{2} g L_{1} \sin \left(\phi_{1}\right) \cos \left(\theta_{1}\right) L_{2} \\
& +\rho_{2} \ddot{L}_{2} \cos \left(\phi_{2}\right) \cos \left(\theta_{2}\right) L_{1} \sin \left(\phi_{1}\right) \cos \left(\theta_{1}\right) L_{2}+\rho_{2} \ddot{L}_{2} \cos \left(\phi_{2}\right) \sin \left(\theta_{2}\right) L_{1} \sin \left(\phi_{1}\right) \sin \left(\theta_{1}\right) L_{2} \\
& +\rho_{2} \dot{L}_{2}^{2} \cos \left(\phi_{2}\right) \sin \left(\theta_{2}\right) L_{1} \sin \left(\phi_{1}\right) \sin \left(\theta_{1}\right)+\rho_{2} \dot{L}_{2}^{2} \cos \left(\phi_{2}\right) \cos \left(\theta_{2}\right) L_{1} \sin \left(\phi_{1}\right) \cos \left(\theta_{1}\right) \\
& +m_{2} \ddot{L}_{2} \cos \left(\phi_{2}\right) \cos \left(\theta_{2}\right) L_{1} \sin \left(\phi_{1}\right) \cos \left(\theta_{1}\right)+m_{2} \ddot{L}_{2} \cos \left(\phi_{2}\right) \sin \left(\theta_{2}\right) L_{1} \sin \left(\phi_{1}\right) \sin \left(\theta_{1}\right) \\
& -m_{2} \ddot{L}_{2} \sin \left(\phi_{2}\right) L_{1} \cos \left(\phi_{1}\right)+Q_{2}+1 / 2 \rho_{1} g \sin \left(\phi_{1}\right) \cos \left(\theta_{1}\right) L_{1}^{2}-\rho_{2} \ddot{L}_{2} \sin \left(\phi_{2}\right) L_{1} \cos \left(\phi_{1}\right) L_{2} \\
& -\rho_{2} \dot{L}_{2}^{2} \sin \left(\phi_{2}\right) L_{1} \cos \left(\phi_{1}\right)
\end{aligned}
$$




$$
\begin{aligned}
& b_{3}=Q_{3}-\rho_{2} \ddot{L}_{1} L_{2}-\rho_{2} \dot{L}_{1} \frac{d}{d t} L_{2}(t)-m_{1} \cos \left(\phi_{1}\right) \cos \left(\theta_{1}\right)-\rho_{1} \ddot{L}_{1} L_{1}-m_{2} \cos \left(\phi_{1}\right) \cos \left(\theta_{1}\right) \\
& -m_{2} \ddot{L}_{2} \sin \left(\phi_{2}\right) \sin \left(\phi_{1}\right)-\rho_{2} \dot{L}_{2}^{2} \sin \left(\phi_{2}\right) \sin \left(\phi_{1}\right) \\
& -\rho_{1} g \cos \left(\phi_{1}\right) \cos \left(\theta_{1}\right) L_{1}-\rho_{2} \dot{L}_{2}^{2} \cos \left(\phi_{2}\right) \cos \left(\theta_{2}\right) \cos \left(\phi_{1}\right) \cos \left(\theta_{1}\right)-\rho_{2} \dot{L}_{2}{ }^{2} \cos \left(\phi_{2}\right) \sin \left(\theta_{2}\right) \cos \left(\phi_{1}\right) \sin \left(\theta_{1}\right) \\
& -m_{2} \ddot{L}_{2} \cos \left(\phi_{2}\right) \cos \left(\theta_{2}\right) \cos \left(\phi_{1}\right) \cos \left(\theta_{1}\right)-m_{2} \ddot{L}_{2} \cos \left(\phi_{2}\right) \sin \left(\theta_{2}\right) \cos \left(\phi_{1}\right) \sin \left(\theta_{1}\right) \\
& -\rho_{2} \ddot{L}_{2} \sin \left(\phi_{2}\right) \sin \left(\phi_{1}\right) L_{2}-\rho_{2} \ddot{L}_{2} \cos \left(\phi_{2}\right) \sin \left(\theta_{2}\right) \cos \left(\phi_{1}\right) \sin \left(\theta_{1}\right) L_{2}-\rho_{2} \ddot{L}_{2} \cos \left(\phi_{2}\right) \cos \left(\theta_{2}\right) \cos \left(\phi_{1}\right) \cos \left(\theta_{1}\right) L_{2} \\
& -m_{2} \ddot{L}_{1}+\left(1 / 2 \rho_{2} L_{2}^{2} \sin \left(\phi_{2}\right) \sin \left(\phi_{1}\right)+m_{2} L_{2} \sin \left(\phi_{2}\right) \sin \left(\phi_{1}\right)+1 / 2 \rho_{2} L_{2}^{2} \cos \left(\phi_{2}\right) \cos \left(\theta_{2}\right) \cos \left(\phi_{1}\right) \cos \left(\theta_{1}\right)\right. \\
& +m_{2} L_{2} \cos \left(\phi_{2}\right) \sin \left(\theta_{2}\right) \cos \left(\phi_{1}\right) \sin \left(\theta_{1}\right)+m_{2} L_{2} \cos \left(\phi_{2}\right) \cos \left(\theta_{2}\right) \cos \left(\phi_{1}\right) \cos \left(\theta_{1}\right) \\
& \left.+1 / 2 \rho_{2} L_{2}{ }^{2} \cos \left(\phi_{2}\right) \sin \left(\theta_{2}\right) \cos \left(\phi_{1}\right) \sin \left(\theta_{1}\right)\right) \dot{\phi}_{2}^{2}+\left(-2 \rho_{2} \dot{L}_{2} \cos \left(\phi_{2}\right) \sin \left(\phi_{1}\right) L_{2}-2 m_{2} \dot{L}_{2} \cos \left(\phi_{2}\right) \sin \left(\phi_{1}\right)\right. \\
& +2 m_{2} \dot{L}_{2} \sin \left(\phi_{2}\right) \sin \left(\theta_{2}\right) \cos \left(\phi_{1}\right) \sin \left(\theta_{1}\right)+2 \rho_{2} \dot{L}_{2} \sin \left(\phi_{2}\right) \sin \left(\theta_{2}\right) \cos \left(\phi_{1}\right) \sin \left(\theta_{1}\right) L_{2} \\
& \left.+2 \rho_{2} \dot{L}_{2} \sin \left(\phi_{2}\right) \cos \left(\theta_{2}\right) \cos \left(\phi_{1}\right) \cos \left(\theta_{1}\right) L_{2}+2 m_{2} \dot{L}_{2} \sin \left(\phi_{2}\right) \cos \left(\theta_{2}\right) \cos \left(\phi_{1}\right) \cos \left(\theta_{1}\right)\right) \dot{\phi}_{2}-1 / 2 \rho_{1} \dot{L}_{1}^{2}-m_{1} \ddot{L}_{1} \\
& +\left(\rho_{2} L_{1}\left(\cos \left(\phi_{1}\right)\right)^{2} L_{2}+m_{2} L_{1}\left(\cos \left(\phi_{1}\right)\right)^{2}+1 / 2 \rho_{1} L_{1}^{2}\left(\cos \left(\phi_{1}\right)\right)^{2}+m_{1} L_{1}\left(\cos \left(\phi_{1}\right)\right)^{2}\right) \dot{\theta}_{1}^{2} \\
& +\left(\rho_{2} L_{1} L_{2}+m_{1} L_{1}+1 / 2 \rho_{1} L_{1}^{2}+m_{2} L_{1}\right) \dot{\phi}_{1}^{2} \\
& +\left(1 / 2 \rho_{2} L_{2}^{2} \cos \left(\phi_{2}\right) \sin \left(\theta_{2}\right) \cos \left(\phi_{1}\right) \sin \left(\theta_{1}\right)+1 / 2 \rho_{2} L_{2}^{2} \cos \left(\phi_{2}\right) \cos \left(\theta_{2}\right) \cos \left(\phi_{1}\right) \cos \left(\theta_{1}\right)\right. \\
& \left.+m_{2} L_{2} \cos \left(\phi_{2}\right) \sin \left(\theta_{2}\right) \cos \left(\phi_{1}\right) \sin \left(\theta_{1}\right)+m_{2} L_{2} \cos \left(\phi_{2}\right) \cos \left(\theta_{2}\right) \cos \left(\phi_{1}\right) \cos \left(\theta_{1}\right)\right) \dot{\theta}_{2}^{2} \\
& +\left(\left(-\rho_{2} L_{2}^{2} \sin \left(\phi_{2}\right) \sin \left(\theta_{2}\right) \cos \left(\phi_{1}\right) \cos \left(\theta_{1}\right)+2 m_{2} L_{2} \sin \left(\phi_{2}\right) \cos \left(\theta_{2}\right) \cos \left(\phi_{1}\right) \sin \left(\theta_{1}\right)\right.\right. \\
& \left.-2 m_{2} L_{2} \sin \left(\phi_{2}\right) \sin \left(\theta_{2}\right) \cos \left(\phi_{1}\right) \cos \left(\theta_{1}\right)+\rho_{2} L_{2}^{2} \sin \left(\phi_{2}\right) \cos \left(\theta_{2}\right) \cos \left(\phi_{1}\right) \sin \left(\theta_{1}\right)\right) \dot{\phi}_{2} \\
& -2 \rho_{2} \dot{L}_{2} \cos \left(\phi_{2}\right) \cos \left(\theta_{2}\right) \cos \left(\phi_{1}\right) \sin \left(\theta_{1}\right) L_{2}-2 m_{2} \dot{L}_{2} \cos \left(\phi_{2}\right) \cos \left(\theta_{2}\right) \cos \left(\phi_{1}\right) \sin \left(\theta_{1}\right) \\
& +2 \rho_{2} \dot{L}_{2} \cos \left(\phi_{2}\right) \sin \left(\theta_{2}\right) \cos \left(\phi_{1}\right) \cos \left(\theta_{1}\right) L_{2}+2 m_{2} \dot{L}_{2} \cos \left(\phi_{2}\right) \sin \left(\theta_{2}\right) \cos \left(\phi_{1}\right) \cos \left(\theta_{1}\right) \dot{\theta}_{2}-\rho_{2} g \cos \left(\phi_{1}\right) \cos \left(\theta_{1}\right) L_{2} \\
& b_{4}=\left(-m_{2} L_{2} \cos \left(\phi_{2}\right) \sin \left(\theta_{2}\right) L_{1} \cos \left(\phi_{1}\right) \cos \left(\theta_{1}\right)+m_{2} L_{2} \cos \left(\phi_{2}\right) \cos \left(\theta_{2}\right) L_{1} \cos \left(\phi_{1}\right) \sin \left(\theta_{1}\right)\right. \\
& \left.-1 / 2 \rho_{2} L_{2}^{2} \cos \left(\phi_{2}\right) \sin \left(\theta_{2}\right) L_{1} \cos \left(\phi_{1}\right) \cos \left(\theta_{1}\right)+1 / 2 \rho_{2} L_{2}^{2} \cos \left(\phi_{2}\right) \cos \left(\theta_{2}\right) L_{1} \cos \left(\phi_{1}\right) \sin \left(\theta_{1}\right)\right) \dot{\phi}_{1}^{2} \\
& +\left(\left(\rho_{2} L_{2}^{2} \cos \left(\phi_{2}\right) \cos \left(\theta_{2}\right) L_{1} \sin \left(\phi_{1}\right) \cos \left(\theta_{1}\right)+\rho_{2} L_{2}^{2} \cos \left(\phi_{2}\right) \sin \left(\theta_{2}\right) L_{1} \sin \left(\phi_{1}\right) \sin \left(\theta_{1}\right)\right.\right. \\
& \left.+2 m_{2} L_{2} \cos \left(\phi_{2}\right) \sin \left(\theta_{2}\right) L_{1} \sin \left(\phi_{1}\right) \sin \left(\theta_{1}\right)+2 m_{2} L_{2} \cos \left(\phi_{2}\right) \cos \left(\theta_{2}\right) L_{1} \sin \left(\phi_{1}\right) \cos \left(\theta_{1}\right)\right) \dot{\theta}_{1} \\
& +2 m_{2} L_{2} \cos \left(\phi_{2}\right) \cos \left(\theta_{2}\right) \dot{L}_{1} \sin \left(\phi_{1}\right) \sin \left(\theta_{1}\right)-\rho_{2} L_{2}^{2} \cos \left(\phi_{2}\right) \sin \left(\theta_{2}\right) \dot{L}_{1} \sin \left(\phi_{1}\right) \cos \left(\theta_{1}\right) \\
& \left.+\rho_{2} L_{2}^{2} \cos \left(\phi_{2}\right) \cos \left(\theta_{2}\right) \dot{L}_{1} \sin \left(\phi_{1}\right) \sin \left(\theta_{1}\right)-2 m_{2} L_{2} \cos \left(\phi_{2}\right) \sin \left(\theta_{2}\right) \dot{L}_{1} \sin \left(\phi_{1}\right) \cos \left(\theta_{1}\right)\right) \dot{\phi}_{1} \\
& +\left(-m_{2} L_{2} \cos \left(\phi_{2}\right) \sin \left(\theta_{2}\right) L_{1} \cos \left(\phi_{1}\right) \cos \left(\theta_{1}\right)+m_{2} L_{2} \cos \left(\phi_{2}\right) \cos \left(\theta_{2}\right) L_{1} \cos \left(\phi_{1}\right) \sin \left(\theta_{1}\right)\right. \\
& -1 / 2 \rho_{2} L_{2}{ }^{2} \cos \left(\phi_{2}\right) \sin \left(\theta_{2}\right) L_{1} \cos \left(\phi_{1}\right) \cos \left(\theta_{1}\right)+1 / 2 \rho_{2} L_{2}^{2} \cos \left(\phi_{2}\right) \cos \left(\theta_{2}\right) L_{1} \cos \left(\phi_{1}\right) \sin \left(\theta_{1}\right) \dot{\theta}_{1}{ }^{2} \\
& +\left(-\rho_{2} L_{2}^{2} \cos \left(\phi_{2}\right) \cos \left(\theta_{2}\right) \dot{L}_{1} \cos \left(\phi_{1}\right) \cos \left(\theta_{1}\right)-2 m_{2} L_{2} \cos \left(\phi_{2}\right) \sin \left(\theta_{2}\right) \dot{L}_{1} \cos \left(\phi_{1}\right) \sin \left(\theta_{1}\right)\right. \\
& \left.-2 m_{2} L_{2} \cos \left(\phi_{2}\right) \cos \left(\theta_{2}\right) \dot{L}_{1} \cos \left(\phi_{1}\right) \cos \left(\theta_{1}\right)-\rho_{2} L_{2}^{2} \cos \left(\phi_{2}\right) \sin \left(\theta_{2}\right) \dot{L}_{1} \cos \left(\phi_{1}\right) \sin \left(\theta_{1}\right)\right) \dot{\theta}_{1} \\
& +\left(\left(2 m_{2} L_{2}^{2} \cos \left(\phi_{2}\right) \sin \left(\phi_{2}\right)+2 / 3 \rho_{2} \cos \left(\phi_{2}\right) L_{2}^{3} \sin \left(\phi_{2}\right)\right) \dot{\phi}_{2}-\rho_{2}\left(\cos \left(\phi_{2}\right)\right)^{2} L_{2}^{2} \dot{L}_{2}\right. \\
& \left.-2 m_{2} L_{2}\left(\cos \left(\phi_{2}\right)\right)^{2} \dot{L}_{2}\right) \dot{\theta}_{2}+m_{2} L_{2} \cos \left(\phi_{2}\right) \sin \left(\theta_{2}\right) \ddot{L}_{1} \cos \left(\phi_{1}\right) \cos \left(\theta_{1}\right) \\
& -1 / 2 \rho_{2} L_{2}^{2} \cos \left(\phi_{2}\right) \cos \left(\theta_{2}\right) \ddot{L}_{1} \cos \left(\phi_{1}\right) \sin \left(\theta_{1}\right)-m_{2} L_{2} \cos \left(\phi_{2}\right) \cos \left(\theta_{2}\right) \ddot{L}_{1} \cos \left(\phi_{1}\right) \sin \left(\theta_{1}\right) \\
& +m_{2} L_{2} \cos \left(\phi_{2}\right) \sin \left(\theta_{2}\right)+1 / 2 \rho_{2} g \cos \left(\phi_{2}\right) \sin \left(\theta_{2}\right) L_{2}^{2}+Q_{4}+1 / 2 \rho_{2} L_{2}{ }^{2} \cos \left(\phi_{2}\right) \sin \left(\theta_{2}\right) \ddot{L}_{1} \cos \left(\phi_{1}\right) \cos \left(\theta_{1}\right)
\end{aligned}
$$




$$
\begin{aligned}
& b_{5}=\left(m_{2} L_{2} \cos \left(\phi_{2}\right) L_{1} \sin \left(\phi_{1}\right)-1 / 2 \rho_{2} L_{2}^{2} \sin \left(\phi_{2}\right) \cos \left(\theta_{2}\right) L_{1} \cos \left(\phi_{1}\right) \cos \left(\theta_{1}\right)-m_{2} L_{2} \sin \left(\phi_{2}\right) \sin \left(\theta_{2}\right) L_{1} \cos \left(\phi_{1}\right) \sin \left(\theta_{1}\right)\right. \\
& -1 / 2 \rho_{2} L_{2}^{2} \sin \left(\phi_{2}\right) \sin \left(\theta_{2}\right) L_{1} \cos \left(\phi_{1}\right) \sin \left(\theta_{1}\right)+1 / 2 \rho_{2} L_{2}^{2} \cos \left(\phi_{2}\right) L_{1} \sin \left(\phi_{1}\right) \\
& \left.-m_{2} L_{2} \sin \left(\phi_{2}\right) \cos \left(\theta_{2}\right) L_{1} \cos \left(\phi_{1}\right) \cos \left(\theta_{1}\right)\right) \dot{\phi}_{1}^{2}+\left(\left(\rho_{2} L_{2}^{2} \sin \left(\phi_{2}\right) \cos \left(\theta_{2}\right) L_{1} \sin \left(\phi_{1}\right) \sin \left(\theta_{1}\right)\right.\right. \\
& +2 m_{2} L_{2} \sin \left(\phi_{2}\right) \cos \left(\theta_{2}\right) L_{1} \sin \left(\phi_{1}\right) \sin \left(\theta_{1}\right)-2 m_{2} L_{2} \sin \left(\phi_{2}\right) \sin \left(\theta_{2}\right) L_{1} \sin \left(\phi_{1}\right) \cos \left(\theta_{1}\right) \\
& \left.-\rho_{2} L_{2}^{2} \sin \left(\phi_{2}\right) \sin \left(\theta_{2}\right) L_{1} \sin \left(\phi_{1}\right) \cos \left(\theta_{1}\right)\right) \dot{\theta}_{1}-\rho_{2} L_{2}{ }^{2} \cos \left(\phi_{2}\right) \dot{L}_{1} \cos \left(\phi_{1}\right)-2 m_{2} L_{2} \cos \left(\phi_{2}\right) \dot{L}_{1} \cos \left(\phi_{1}\right) \\
& -\rho_{2} L_{2}^{2} \sin \left(\phi_{2}\right) \cos \left(\theta_{2}\right) \dot{L}_{1} \sin \left(\phi_{1}\right) \cos \left(\theta_{1}\right)-\rho_{2} L_{2}^{2} \sin \left(\phi_{2}\right) \sin \left(\theta_{2}\right) \dot{L}_{1} \sin \left(\phi_{1}\right) \sin \left(\theta_{1}\right) \\
& \left.-2 m_{2} L_{2} \sin \left(\phi_{2}\right) \cos \left(\theta_{2}\right) \dot{L}_{1} \sin \left(\phi_{1}\right) \cos \left(\theta_{1}\right)-2 m_{2} L_{2} \sin \left(\phi_{2}\right) \sin \left(\theta_{2}\right) \dot{L}_{1} \sin \left(\phi_{1}\right) \sin \left(\theta_{1}\right)\right) \dot{\phi}_{1} \\
& +\left(-1 / 2 \rho_{2} L_{2}^{2} \sin \left(\phi_{2}\right) \sin \left(\theta_{2}\right) L_{1} \cos \left(\phi_{1}\right) \sin \left(\theta_{1}\right)-m_{2} L_{2} \sin \left(\phi_{2}\right) \cos \left(\theta_{2}\right) L_{1} \cos \left(\phi_{1}\right) \cos \left(\theta_{1}\right)\right. \\
& -m_{2} L_{2} \sin \left(\phi_{2}\right) \sin \left(\theta_{2}\right) L_{1} \cos \left(\phi_{1}\right) \sin \left(\theta_{1}\right)-1 / 2 \rho_{2} L_{2}^{2} \sin \left(\phi_{2}\right) \cos \left(\theta_{2}\right) L_{1} \cos \left(\phi_{1}\right) \cos \left(\theta_{1}\right) \dot{\theta}_{1}^{2} \\
& +\left(-\rho_{2} L_{2}^{2} \sin \left(\phi_{2}\right) \cos \left(\theta_{2}\right) \dot{L}_{1} \cos \left(\phi_{1}\right) \sin \left(\theta_{1}\right)+2 m_{2} L_{2} \sin \left(\phi_{2}\right) \sin \left(\theta_{2}\right) \dot{L}_{1} \cos \left(\phi_{1}\right) \cos \left(\theta_{1}\right)\right. \\
& +\rho_{2} L_{2}{ }^{2} \sin \left(\phi_{2}\right) \sin \left(\theta_{2}\right) \dot{L}_{1} \cos \left(\phi_{1}\right) \cos \left(\theta_{1}\right)-2 m_{2} L_{2} \sin \left(\phi_{2}\right) \cos \left(\theta_{2}\right) \dot{L}_{1} \cos \left(\phi_{1}\right) \sin \left(\theta_{1}\right) \dot{\theta}_{1} \\
& +\left(-1 / 3 \rho_{2} \cos \left(\phi_{2}\right) L_{2}^{3} \sin \left(\phi_{2}\right)-m_{2} L_{2}^{2} \cos \left(\phi_{2}\right) \sin \left(\phi_{2}\right)\right) \dot{\theta}_{2}^{2}+\left(-\rho_{2} L_{2}^{2} \dot{L}_{2}-2 m_{2} L_{2} \dot{L}_{2}\right) \dot{\phi}_{2} \\
& +1 / 2 \rho_{2} L_{2}^{2} \sin \left(\phi_{2}\right) \sin \left(\theta_{2}\right) \ddot{L}_{1} \cos \left(\phi_{1}\right) \sin \left(\theta_{1}\right)-1 / 2 \rho_{2} L_{2}^{2} \cos \left(\phi_{2}\right) \ddot{L}_{1} \sin \left(\phi_{1}\right) \\
& +1 / 2 \rho_{2} g \sin \left(\phi_{2}\right) \cos \left(\theta_{2}\right) L_{2}^{2}+m_{2} L_{2} \sin \left(\phi_{2}\right) \cos \left(\theta_{2}\right) \ddot{L}_{1} \cos \left(\phi_{1}\right) \cos \left(\theta_{1}\right)-m_{2} L_{2} \cos \left(\phi_{2}\right) \ddot{L}_{1} \sin \left(\phi_{1}\right) \\
& +Q_{5}+1 / 2 \rho_{2} L_{2}^{2} \sin \left(\phi_{2}\right) \cos \left(\theta_{2}\right) \ddot{L}_{1} \cos \left(\phi_{1}\right) \cos \left(\theta_{1}\right)+m_{2} L_{2} \sin \left(\phi_{2}\right) \sin \left(\theta_{2}\right) \ddot{L}_{1} \cos \left(\phi_{1}\right) \sin \left(\theta_{1}\right) \\
& +m_{2} L_{2} \sin \left(\phi_{2}\right) \cos \left(\theta_{2}\right) \\
& b_{6}=\left(m_{2} \sin \left(\phi_{2}\right) L_{1} \sin \left(\phi_{1}\right)+m_{2} \cos \left(\phi_{2}\right) \sin \left(\theta_{2}\right) L_{1} \cos \left(\phi_{1}\right) \sin \left(\theta_{1}\right)+\rho_{2} \cos \left(\phi_{2}\right) \cos \left(\theta_{2}\right) L_{1} \cos \left(\phi_{1}\right) \cos \left(\theta_{1}\right) L_{2}\right. \\
& +\rho_{2} \cos \left(\phi_{2}\right) \sin \left(\theta_{2}\right) L_{1} \cos \left(\phi_{1}\right) \sin \left(\theta_{1}\right) L_{2}+m_{2} \cos \left(\phi_{2}\right) \cos \left(\theta_{2}\right) L_{1} \cos \left(\phi_{1}\right) \cos \left(\theta_{1}\right)+\rho_{2} \sin \left(\phi_{2}\right) L_{1} \sin \left(\phi_{1}\right) L_{2} \\
& \left.+1 / 2 \rho_{2} L_{1}^{2}\right) \dot{\phi}_{1}^{2}+\left(\left(-2 \rho_{2} \cos \left(\phi_{2}\right) \cos \left(\theta_{2}\right) L_{1} \sin \left(\phi_{1}\right) \sin \left(\theta_{1}\right) L_{2}+2 m_{2} \cos \left(\phi_{2}\right) \sin \left(\theta_{2}\right) L_{1} \sin \left(\phi_{1}\right) \cos \left(\theta_{1}\right)\right.\right. \\
& \left.-2 m_{2} \cos \left(\phi_{2}\right) \cos \left(\theta_{2}\right) L_{1} \sin \left(\phi_{1}\right) \sin \left(\theta_{1}\right)+2 \rho_{2} \cos \left(\phi_{2}\right) \sin \left(\theta_{2}\right) L_{1} \sin \left(\phi_{1}\right) \cos \left(\theta_{1}\right) L_{2}\right) \dot{\theta}_{1} \\
& -2 \rho_{2} \sin \left(\phi_{2}\right) \dot{L}_{1} \cos \left(\phi_{1}\right) L_{2}+2 m_{2} \cos \left(\phi_{2}\right) \cos \left(\theta_{2}\right) \dot{L}_{1} \sin \left(\phi_{1}\right) \cos \left(\theta_{1}\right) \\
& -2 m_{2} \sin \left(\phi_{2}\right) \dot{L}_{1} \cos \left(\phi_{1}\right)+2 \rho_{2} \cos \left(\phi_{2}\right) \cos \left(\theta_{2}\right) \dot{L}_{1} \sin \left(\phi_{1}\right) \cos \left(\theta_{1}\right) L_{2} \\
& \left.+2 \rho_{2} \cos \left(\phi_{2}\right) \sin \left(\theta_{2}\right) \dot{L}_{1} \sin \left(\phi_{1}\right) \sin \left(\theta_{1}\right) L_{2}+2 m_{2} \cos \left(\phi_{2}\right) \sin \left(\theta_{2}\right) \dot{L}_{1} \sin \left(\phi_{1}\right) \sin \left(\theta_{1}\right)\right) \dot{\phi}_{1} \\
& +\left(\rho_{2} \cos \left(\phi_{2}\right) \sin \left(\theta_{2}\right) L_{1} \cos \left(\phi_{1}\right) \sin \left(\theta_{1}\right) L_{2}+m_{2} \cos \left(\phi_{2}\right) \cos \left(\theta_{2}\right) L_{1} \cos \left(\phi_{1}\right) \cos \left(\theta_{1}\right)\right. \\
& +m_{2} \cos \left(\phi_{2}\right) \sin \left(\theta_{2}\right) L_{1} \cos \left(\phi_{1}\right) \sin \left(\theta_{1}\right)+\rho_{2} \cos \left(\phi_{2}\right) \cos \left(\theta_{2}\right) L_{1} \cos \left(\phi_{1}\right) \cos \left(\theta_{1}\right) L_{2} \\
& \left.+1 / 2 \rho_{2} L_{1}^{2}\left(\cos \left(\phi_{1}\right)\right)^{2}\right) \dot{\theta}_{1}^{2}+\left(2 \rho_{2} \cos \left(\phi_{2}\right) \cos \left(\theta_{2}\right) \dot{L}_{1} \cos \left(\phi_{1}\right) \sin \left(\theta_{1}\right) L_{2}\right. \\
& -2 m_{2} \cos \left(\phi_{2}\right) \sin \left(\theta_{2}\right) \dot{L}_{1} \cos \left(\phi_{1}\right) \cos \left(\theta_{1}\right)-2 \rho_{2} \cos \left(\phi_{2}\right) \sin \left(\theta_{2}\right) \dot{L}_{1} \cos \left(\phi_{1}\right) \cos \left(\theta_{1}\right) L_{2} \\
& +2 m_{2} \cos \left(\phi_{2}\right) \cos \left(\theta_{2}\right) \dot{L}_{1} \cos \left(\phi_{1}\right) \sin \left(\theta_{1}\right) \dot{\theta}_{1}+\left(1 / 2 \rho_{2} L_{2}{ }^{2}\left(\cos \left(\phi_{2}\right)\right)^{2}+m_{2} L_{2}\left(\cos \left(\phi_{2}\right)\right)^{2}\right) \dot{\theta}_{2}^{2} \\
& +\left(1 / 2 \rho_{2} L_{2}^{2}+m_{2} L_{2}\right) \dot{\phi}_{2}^{2}-\rho_{2} \ddot{L}_{2}-m_{2} \ddot{L}_{2}-m_{2} \cos \left(\phi_{2}\right) \cos \left(\theta_{2}\right)-1 / 2 \rho_{2} \dot{L}_{2}{ }^{2} \\
& -\rho_{2} g L_{1} \cos \left(\phi_{1}\right) \cos \left(\theta_{1}\right)-\rho_{2} \cos \left(\phi_{2}\right) \cos \left(\theta_{2}\right) \ddot{L}_{1} \cos \left(\phi_{1}\right) \cos \left(\theta_{1}\right) L_{2}-\rho_{2} \cos \left(\phi_{2}\right) \sin \left(\theta_{2}\right) \ddot{L}_{1} \cos \left(\phi_{1}\right) \sin \left(\theta_{1}\right) L_{2} \\
& -\rho_{2} \sin \left(\phi_{2}\right) \ddot{L}_{1} \sin \left(\phi_{1}\right) L_{2}-\rho_{2} g L_{2} \cos \left(\phi_{2}\right) \cos \left(\theta_{2}\right)-m_{2} \cos \left(\phi_{2}\right) \cos \left(\theta_{2}\right) \ddot{L}_{1} \cos \left(\phi_{1}\right) \cos \left(\theta_{1}\right) \\
& -m_{2} \sin \left(\phi_{2}\right) \ddot{L}_{1} \sin \left(\phi_{1}\right)+1 / 2 \rho_{2} \dot{L}_{1}^{2}-m_{2} \cos \left(\phi_{2}\right) \sin \left(\theta_{2}\right) \ddot{L}_{1} \cos \left(\phi_{1}\right) \sin \left(\theta_{1}\right)+Q_{6}
\end{aligned}
$$

The generalized forces denoted by $Q_{j}$ are obtained via the principle of virtual work as follows

$$
Q_{q_{j}}=\sum_{j=1}^{6} \sum_{i=1}^{N} \boldsymbol{F}_{i} \cdot \frac{\partial \boldsymbol{R}_{i}}{\partial q_{j}}
$$

Note that this shows that when relative generalized coordinates are used the generalized forces for the $N$ th tether contain contributions of all preceding tether forces. The key external forces on the system are tether drag and aerodynamic forces on the kite. These are derived in Section $\mathrm{C}$ below.

\section{B. Multiple-Kites on Different Lines}

The alternative system configuration is modeled as illustrated in Fig. 3. An element of tether on the main power line has a position given by 


$$
\boldsymbol{R}_{0}\left(s_{0}\right)=s_{0} \cos \phi_{0} \sin \theta_{0} \boldsymbol{i}+s_{0} \sin \phi_{0} \boldsymbol{j}+s_{0} \cos \phi_{0} \cos \theta_{0} \boldsymbol{k}
$$

The inertial position of a point on the first tether branch can be written as

$$
\boldsymbol{R}_{j}\left(s_{j}\right)=\boldsymbol{r}_{j}\left(s_{j}\right)+\sum_{k=1}^{j-1} \boldsymbol{r}_{k}\left(L_{k}\right)+\boldsymbol{R}_{0}\left(L_{0}\right)
$$

Similarly, for the second tether branch we have

$$
\boldsymbol{R}_{j}\left(s_{j}\right)=\boldsymbol{r}_{j}\left(s_{j}\right)+\sum_{k=n+1}^{j-1} \boldsymbol{r}_{k}\left(L_{k}\right)+\boldsymbol{R}_{0}\left(L_{0}\right)
$$

The kinetic and potential energies of the system is given by the sum of the contribution from all tethers and kites

$$
\begin{gathered}
\mathcal{T}=\frac{1}{2} \rho_{c_{0}} \int_{0}^{L_{0}} \dot{\boldsymbol{R}}_{0}\left(s_{0}\right) \cdot \dot{\boldsymbol{R}}_{0}\left(s_{0}\right) \mathrm{d} s_{0}+\sum_{j=1}^{2 n} \frac{1}{2} \rho_{c_{j}} \int_{0}^{L_{j}} \dot{\boldsymbol{R}}_{j}\left(s_{j}\right) \cdot \dot{\boldsymbol{R}}_{j}\left(s_{j}\right) \mathrm{d} s_{j}+\left.\frac{1}{2} m_{k_{j}} \dot{\boldsymbol{R}}_{j}\left(s_{j}\right) \cdot \dot{\boldsymbol{R}}_{j}\left(s_{j}\right)\right|_{s_{j}=L_{j}} \\
\mathcal{V}=\sum_{j=1}^{2 n} \rho_{c_{j}} g \int_{0}^{L_{j}} \boldsymbol{R}_{j}\left(s_{j}\right) \cdot \boldsymbol{k} \mathrm{d} s_{j}+\left.m_{k_{j}} g \boldsymbol{R}_{j}\left(s_{j}\right) \cdot \boldsymbol{k}\right|_{s_{j}=L_{j}}
\end{gathered}
$$

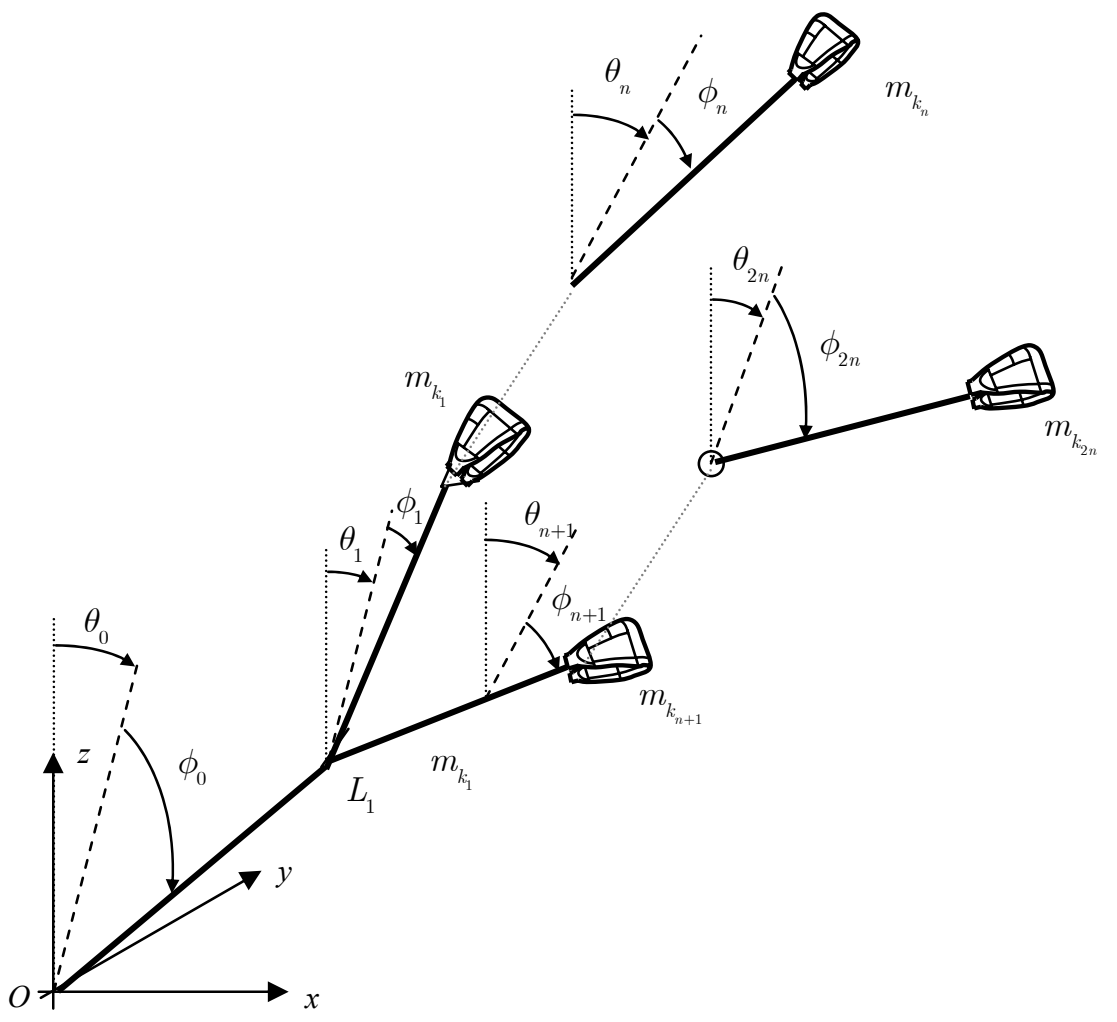

Fig. 3 Multiple kite model with kites connected on branched lines.

1. Two-Kite System

Using Lagrange's equations the equations of motion can be expressed in the form

$$
[\boldsymbol{A}] \boldsymbol{q}=\boldsymbol{b}
$$

where $\boldsymbol{q}=\left[\ddot{\theta}_{0}, \ddot{\phi}_{0}, T_{0}, \ddot{\theta}_{1}, \ddot{\phi}_{1}, T_{1}, \ddot{\theta}_{2}, \ddot{\phi}_{2}, T_{2}\right]$ is a pseudo-acceleration vector. The elements of the system matrix are 


$$
\boldsymbol{A}=\left[\begin{array}{ccccccccc}
A_{1,1} & 0 & 0 & A_{1,4} & A_{1,5} & 0 & A_{1,7} & A_{1,8} & 0 \\
0 & A_{2,2} & 0 & A_{2,4} & A_{2,5} & 0 & A_{2,7} & A_{2,8} & 0 \\
0 & 0 & 1 & A_{3,4} & A_{3,5} & 0 & A_{3,7} & A_{3,8} & 0 \\
A_{1,4} & A_{2,4} & 0 & A_{4,4} & 0 & 0 & 0 & 0 & 0 \\
A_{1,5} & A_{2,5} & 0 & 0 & A_{5,5} & 0 & 0 & 0 & 0 \\
A_{6,1} & A_{6,2} & 0 & 0 & 0 & 1 & 0 & 0 & 0 \\
A_{1,7} & A_{2,7} & 0 & 0 & 0 & 0 & A_{7,7} & 0 & 0 \\
A_{1,8} & A_{2,8} & 0 & 0 & 0 & 0 & 0 & A_{8,8} & 0 \\
A_{9,1} & A_{9,2} & 0 & 0 & 0 & 0 & 0 & 0 & 1
\end{array}\right]
$$

where

$$
\begin{aligned}
& A_{1,1}=1 / 3 L_{0}^{2} \cos ^{2} \phi_{0}\left(\rho_{0} L_{0}+3 m_{2}+3 L_{1} \rho_{1}+3 L_{2} \rho_{2}+3 m_{1}\right) \\
& A_{1,4}=1 / 2 L_{0} L_{1} \cos \phi_{1} \cos \phi_{0}\left(\sin \theta_{1} \sin \theta_{0}+\cos \theta_{1} \cos \theta_{0}\right)\left(L_{1} \rho_{1}+2 m_{1}\right) \\
& A_{1,5}=-1 / 2 L_{0} L_{1} \sin \phi_{1} \cos \phi_{0}\left(\sin \theta_{1} \cos \theta_{0}-\cos \theta_{1} \sin \theta_{0}\right)\left(L_{1} \rho_{1}+2 m_{1}\right) \\
& A_{1,7}=1 / 2 L_{0} L_{2} \cos \phi_{2} \cos \phi_{0}\left(\sin \theta_{2} \sin \theta_{0}+\cos \theta_{0} \cos \theta_{2}\right)\left(L_{2} \rho_{2}+2 m_{2}\right) \\
& A_{1,8}=-1 / 2 L_{0} L_{2} \sin \phi_{2} \cos \phi_{0}\left(\sin \theta_{2} \cos \theta_{0}-\sin \theta_{0} \cos \theta_{2}\right)\left(L_{2} \rho_{2}+2 m_{2}\right) \\
& A_{2,2}=1 / 3 L_{0}^{2}\left(\rho_{0} L_{0}+3 m_{2}+3 L_{1} \rho_{1}+3 L_{2} \rho_{2}+3 m_{1}\right) \\
& A_{2,4}=1 / 2 L_{0} L_{1} \cos \phi_{1} \sin \phi_{0}\left(\sin \theta_{1} \cos \theta_{0}-\cos \theta_{1} \sin \theta_{0}\right)\left(L_{1} \rho_{1}+2 m_{1}\right) \\
& A_{2,5}=1 / 2 L_{0} L_{1}\left(\sin \phi_{1} \sin \theta_{1} \sin \phi_{0} \sin \theta_{0}+\cos \phi_{1} \cos \phi_{0}+\sin \phi_{1} \cos \theta_{1} \sin \phi_{0} \cos \theta_{0}\right)\left(L_{1} \rho_{1}+2 m_{1}\right) \\
& A_{2,7}=1 / 2 L_{0} L_{2} \sin \phi_{0} \cos \phi_{2}\left(\sin \theta_{2} \cos \theta_{0}-\sin \theta_{0} \cos \theta_{2}\right)\left(L_{2} \rho_{2}+2 m_{2}\right) \\
& A_{2,8}=1 / 2 L_{0} L_{2}\left(\sin \phi_{2} \cos \theta_{2} \sin \phi_{0} \cos \theta_{0}+\sin \phi_{2} \sin \theta_{2} \sin \phi_{0} \sin \theta_{0}+\cos \phi_{2} \cos \phi_{0}\right)\left(L_{2} \rho_{2}+2 m_{2}\right) \\
& A_{3,4}=1 / 2 L_{1} \cos \phi_{1} \cos \phi_{0}\left(\cos \theta_{1} \sin \theta_{0}-\cos \theta_{0} \sin \theta_{1}\right)\left(L_{1} \rho_{1}+2 m_{1}\right) \\
& A_{3,5}=-1 / 2 L_{1}\left(\sin \phi_{1} \sin \theta_{1} \cos \phi_{0} \sin \theta_{0}-\cos \phi_{1} \sin \phi_{0}+\sin \phi_{1} \cos \theta_{1} \cos \phi_{0} \cos \theta_{0}\right)\left(L_{1} \rho_{1}+2 m_{1}\right) \\
& A_{3,7}=-1 / 2 L_{2} \cos \phi_{0} \cos \phi_{2}\left(\sin \theta_{2} \cos \theta_{0}-\cos \theta_{2} \sin \theta_{0}\right)\left(L_{2} \rho_{2}+2 m_{2}\right) \\
& A_{3,8}=1 / 2 L_{2}\left(-\sin \phi_{2} \sin \theta_{2} \cos \phi_{0} \sin \theta_{0}+\cos \phi_{2} \sin \phi_{0}-\sin \phi_{2} \cos \theta_{2} \cos \phi_{0} \cos \theta_{0}\right)\left(L_{2} \rho_{2}+2 m_{2}\right) \\
& A_{4,4}=1 / 3 L_{1}^{2} \cos ^{2} \phi_{1}\left(L_{1} \rho_{1}+3 m_{1}\right) \\
& A_{5,5}=1 / 3 L_{1}^{2}\left(L_{1} \rho_{1}+3 m_{1}\right) \\
& A_{6,1}=-L_{0} \cos \phi_{1} \cos \phi_{0}\left(\cos \theta_{1} \sin \theta_{0}-\cos \theta_{0} \sin \theta_{1}\right)\left(L_{1} \rho_{1}+m_{1}\right) \\
& A_{6,2}=L_{0}\left(\sin \phi_{1} \cos \phi_{0}-\cos \phi_{1} \sin \theta_{1} \sin \phi_{0} \sin \theta_{0}-\cos \phi_{1} \cos \theta_{1} \sin \phi_{0} \cos \theta_{0}\right)\left(L_{1} \rho_{1}+m_{1}\right) \\
& A_{7,7}=1 / 3 L_{2}^{2} \cos ^{2} \phi_{2}\left(L_{2} \rho_{2}+3 m_{2}\right) \\
& A_{8,8}=1 / 3 L_{2}^{2}\left(L_{2} \rho_{2}+3 m_{2}\right) \\
& A_{9,1}=L_{0} \cos \phi_{2} \cos \phi_{0}\left(\sin \theta_{2} \cos \theta_{0}-\cos \theta_{2} \sin \theta_{0}\right)\left(L_{2} \rho_{2}+m_{2}\right)
\end{aligned}
$$




$$
A_{9,2}=L_{0}\left(\sin \phi_{2} \cos \phi_{0}-\cos \phi_{2} \sin \theta_{2} \sin \phi_{0} \sin \theta_{0}-\cos \phi_{2} \cos \theta_{2} \sin \phi_{0} \cos \theta_{0}\right)\left(L_{2} \rho_{2}+m_{2}\right)
$$

\section{The right-hand side of the equations of motion are given by}

$$
\begin{aligned}
& b_{1}=2 m_{2} L_{2} \sin \left(\phi_{2}\right) \dot{\phi}_{2} \cos \left(\theta_{2}\right) \dot{\theta}_{2} L_{0} \cos \left(\phi_{0}\right) \cos \left(\theta_{0}\right)-2 \rho_{1} L_{0}\left(\cos \left(\phi_{0}\right)\right)^{2} \dot{\theta}_{0} L_{1} \dot{L}_{0} \\
& -\rho_{1} \dot{L}_{1}^{2} \cos \left(\phi_{1}\right) \sin \left(\theta_{1}\right) L_{0} \cos \left(\phi_{0}\right) \cos \left(\theta_{0}\right)-2 \rho_{1} \dot{L}_{1} \cos \left(\phi_{1}\right) \sin \left(\theta_{1}\right) \dot{\theta}_{1} L_{0} \cos \left(\phi_{0}\right) \sin \left(\theta_{0}\right) L_{1} \\
& +\rho_{1} \dot{L}_{1}^{2} \cos \left(\phi_{1}\right) \cos \left(\theta_{1}\right) L_{0} \cos \left(\phi_{0}\right) \sin \left(\theta_{0}\right)+\rho_{1} L_{1}^{2} \sin \left(\phi_{1}\right) \dot{\phi}_{1} \sin \left(\theta_{1}\right) \dot{\theta}_{1} L_{0} \cos \left(\phi_{0}\right) \sin \left(\theta_{0}\right) \\
& +\rho_{1} L_{1}^{2} \sin \left(\phi_{1}\right) \dot{\phi}_{1} \cos \left(\theta_{1}\right) \dot{\theta}_{1} L_{0} \cos \left(\phi_{0}\right) \cos \left(\theta_{0}\right)-1 / 2 \rho_{1} L_{1}^{2} \cos \left(\phi_{1}\right) \dot{\phi}_{1}^{2} \cos \left(\theta_{1}\right) L_{0} \cos \left(\phi_{0}\right) \sin \left(\theta_{0}\right) \\
& +2 \rho_{2} \dot{L}_{2} \sin \left(\phi_{2}\right) \dot{\phi}_{2} \sin \left(\theta_{2}\right) L_{0} \cos \left(\phi_{0}\right) \cos \left(\theta_{0}\right) L_{2}+2 \rho_{2} L_{0}{ }^{2} \cos \left(\phi_{0}\right) \dot{\theta}_{0} L_{2} \sin \left(\phi_{0}\right) \dot{\phi}_{0} \\
& -2 \rho_{2} \dot{L}_{2} \cos \left(\phi_{2}\right) \cos \left(\theta_{2}\right) \dot{\theta}_{2} L_{0} \cos \left(\phi_{0}\right) \cos \left(\theta_{0}\right) L_{2}-\rho_{2} \dot{L}_{2}^{2} \cos \left(\phi_{2}\right) \sin \left(\theta_{2}\right) L_{0} \cos \left(\phi_{0}\right) \cos \left(\theta_{0}\right) \\
& -2 \rho_{2} \dot{L}_{2} \sin \left(\phi_{2}\right) \dot{\phi}_{2} \cos \left(\theta_{2}\right) L_{0} \cos \left(\phi_{0}\right) \sin \left(\theta_{0}\right) L_{2}-2 \rho_{2} \dot{L}_{2} \cos \left(\phi_{2}\right) \sin \left(\theta_{2}\right) \dot{\theta}_{2} L_{0} \cos \left(\phi_{0}\right) \sin \left(\theta_{0}\right) L_{2} \\
& +\rho_{2} \dot{L}_{2}^{2} \cos \left(\phi_{2}\right) \cos \left(\theta_{2}\right) L_{0} \cos \left(\phi_{0}\right) \sin \left(\theta_{0}\right)+\rho_{2} L_{2}^{2} \sin \left(\phi_{2}\right) \dot{\phi}_{2} \cos \left(\theta_{2}\right) \dot{\theta}_{2} L_{0} \cos \left(\phi_{0}\right) \cos \left(\theta_{0}\right) \\
& +\rho_{2} L_{2}^{2} \sin \left(\phi_{2}\right) \dot{\phi}_{2} \sin \left(\theta_{2}\right) \dot{\theta}_{2} L_{0} \cos \left(\phi_{0}\right) \sin \left(\theta_{0}\right)+2 \rho_{1} L_{0}^{2} \cos \left(\phi_{0}\right) \dot{\theta}_{0} L_{1} \sin \left(\phi_{0}\right) \dot{\phi}_{0} \\
& -1 / 2 \rho_{2} L_{2}^{2} \cos \left(\phi_{2}\right) \cos \left(\theta_{2}\right) \dot{\theta}_{2}^{2} L_{0} \cos \left(\phi_{0}\right) \sin \left(\theta_{0}\right)+2 m_{1} L_{1} \sin \left(\phi_{1}\right) \dot{\phi}_{1} \sin \left(\theta_{1}\right) \dot{\theta}_{1} L_{0} \cos \left(\phi_{0}\right) \sin \left(\theta_{0}\right) \\
& -2 m_{1} L_{0}\left(\cos \left(\phi_{0}\right)\right)^{2} \dot{\theta}_{0} \dot{L}_{0}+m_{1} L_{1} \cos \left(\phi_{1}\right) \dot{\phi}_{1}^{2} \sin \left(\theta_{1}\right) L_{0} \cos \left(\phi_{0}\right) \cos \left(\theta_{0}\right) \\
& +2 m_{1} L_{1} \sin \left(\phi_{1}\right) \dot{\phi}_{1} \cos \left(\theta_{1}\right) \dot{\theta}_{1} L_{0} \cos \left(\phi_{0}\right) \cos \left(\theta_{0}\right)-2 m_{1} \dot{L}_{1} \cos \left(\phi_{1}\right) \cos \left(\theta_{1}\right) \dot{\theta}_{1} L_{0} \cos \left(\phi_{0}\right) \cos \left(\theta_{0}\right) \\
& +m_{1} L_{1} \cos \left(\phi_{1}\right) \sin \left(\theta_{1}\right) \dot{\theta}_{1}^{2} L_{0} \cos \left(\phi_{0}\right) \cos \left(\theta_{0}\right)-\rho_{2} L_{0}^{2}\left(\cos \left(\phi_{0}\right)\right)^{2} \dot{\theta}_{0} \dot{L}_{2} \\
& -2 m_{1} \dot{L}_{1} \sin \left(\phi_{1}\right) \dot{\phi}_{1} \cos \left(\theta_{1}\right) L_{0} \cos \left(\phi_{0}\right) \sin \left(\theta_{0}\right)-m_{1} L_{1} \cos \left(\phi_{1}\right) \dot{\phi}_{1}^{2} \cos \left(\theta_{1}\right) L_{0} \cos \left(\phi_{0}\right) \sin \left(\theta_{0}\right) \\
& +m_{1} L_{0} \cos \left(\phi_{0}\right) \sin \left(\theta_{0}\right)+m_{2} L_{0} \cos \left(\phi_{0}\right) \sin \left(\theta_{0}\right)+Q_{1}+2 m_{2} L_{2} \sin \left(\phi_{2}\right) \dot{\phi}_{2} \sin \left(\theta_{2}\right) \dot{\theta}_{2} L_{0} \cos \left(\phi_{0}\right) \sin \left(\theta_{0}\right) \\
& +2 m_{2} L_{0}^{2} \cos \left(\phi_{0}\right) \dot{\theta}_{0} \sin \left(\phi_{0}\right) \dot{\phi}_{0}-2 m_{2} L_{0}\left(\cos \left(\phi_{0}\right)\right)^{2} \dot{\theta}_{0} \dot{L}_{0}-2 m_{2} \dot{L}_{2} \cos \left(\phi_{2}\right) \sin \left(\theta_{2}\right) \dot{\theta}_{2} L_{0} \cos \left(\phi_{0}\right) \sin \left(\theta_{0}\right) \\
& -m_{2} L_{2} \cos \left(\phi_{2}\right) \cos \left(\theta_{2}\right) \dot{\theta}_{2}^{2} L_{0} \cos \left(\phi_{0}\right) \sin \left(\theta_{0}\right)+2 m_{2} \dot{L}_{2} \sin \left(\phi_{2}\right) \dot{\phi}_{2} \sin \left(\theta_{2}\right) L_{0} \cos \left(\phi_{0}\right) \cos \left(\theta_{0}\right) \\
& -2 m_{2} \dot{L}_{2} \cos \left(\phi_{2}\right) \cos \left(\theta_{2}\right) \dot{\theta}_{2} L_{0} \cos \left(\phi_{0}\right) \cos \left(\theta_{0}\right)-2 m_{2} \dot{L}_{2} \sin \left(\phi_{2}\right) \dot{\phi}_{2} \cos \left(\theta_{2}\right) L_{0} \cos \left(\phi_{0}\right) \sin \left(\theta_{0}\right) \\
& -m_{2} L_{2} \cos \left(\phi_{2}\right) \dot{\phi}_{2}^{2} \cos \left(\theta_{2}\right) L_{0} \cos \left(\phi_{0}\right) \sin \left(\theta_{0}\right)+m_{2} L_{2} \cos \left(\phi_{2}\right) \sin \left(\theta_{2}\right) \dot{\theta}_{2}^{2} L_{0} \cos \left(\phi_{0}\right) \cos \left(\theta_{0}\right) \\
& +\rho_{2} g L_{0} \cos \left(\phi_{0}\right) \sin \left(\theta_{0}\right) L_{2}+1 / 2 \rho_{0} g \cos \left(\phi_{0}\right) \sin \left(\theta_{0}\right) L_{0}{ }^{2}-1 / 2 \rho_{2} L_{2}{ }^{2} \cos \left(\phi_{2}\right) \dot{\phi}_{2}{ }^{2} \cos \left(\theta_{2}\right) L_{0} \cos \left(\phi_{0}\right) \sin \left(\theta_{0}\right) \\
& +2 m_{1} \dot{L}_{1} \sin \left(\phi_{1}\right) \dot{\phi}_{1} \sin \left(\theta_{1}\right) L_{0} \cos \left(\phi_{0}\right) \cos \left(\theta_{0}\right)+1 / 2 \rho_{2} L_{2}^{2} \cos \left(\phi_{2}\right) \dot{\phi}_{2}^{2} \sin \left(\theta_{2}\right) L_{0} \cos \left(\phi_{0}\right) \cos \left(\theta_{0}\right) \\
& +1 / 2 \rho_{2} L_{2}^{2} \cos \left(\phi_{2}\right) \sin \left(\theta_{2}\right) \dot{\theta}_{2}^{2} L_{0} \cos \left(\phi_{0}\right) \cos \left(\theta_{0}\right)-2 \rho_{2} L_{0}\left(\cos \left(\phi_{0}\right)\right)^{2} \dot{\theta}_{0} L_{2} \dot{L}_{0}-\rho_{0}\left(\cos \left(\phi_{0}\right)\right)^{2} \dot{\theta}_{0} L_{0}^{2} \dot{L}_{0} \\
& +2 m_{1} L_{0}^{2} \cos \left(\phi_{0}\right) \dot{\theta}_{0} \sin \left(\phi_{0}\right) \dot{\phi}_{0}+m_{2} L_{2} \cos \left(\phi_{2}\right) \dot{\phi}_{2}^{2} \sin \left(\theta_{2}\right) L_{0} \cos \left(\phi_{0}\right) \cos \left(\theta_{0}\right) \\
& -1 / 2 \rho_{1} L_{1}^{2} \cos \left(\phi_{1}\right) \cos \left(\theta_{1}\right) \dot{\theta}_{1}^{2} L_{0} \cos \left(\phi_{0}\right) \sin \left(\theta_{0}\right)+1 / 2 \rho_{1} L_{1}^{2} \cos \left(\phi_{1}\right) \sin \left(\theta_{1}\right) \dot{\theta}_{1}^{2} L_{0} \cos \left(\phi_{0}\right) \cos \left(\theta_{0}\right) \\
& -2 m_{1} \dot{L}_{1} \cos \left(\phi_{1}\right) \sin \left(\theta_{1}\right) \dot{\theta}_{1} L_{0} \cos \left(\phi_{0}\right) \sin \left(\theta_{0}\right)-2 \rho_{1} \dot{L}_{1} \sin \left(\phi_{1}\right) \dot{\phi}_{1} \cos \left(\theta_{1}\right) L_{0} \cos \left(\phi_{0}\right) \sin \left(\theta_{0}\right) L_{1} \\
& -2 \rho_{1} \dot{L}_{1} \cos \left(\phi_{1}\right) \cos \left(\theta_{1}\right) \dot{\theta}_{1} L_{0} \cos \left(\phi_{0}\right) \cos \left(\theta_{0}\right) L_{1}+2 \rho_{1} \dot{L}_{1} \sin \left(\phi_{1}\right) \dot{\phi}_{1} \sin \left(\theta_{1}\right) L_{0} \cos \left(\phi_{0}\right) \cos \left(\theta_{0}\right) L_{1} \\
& -\rho_{1} L_{0}^{2}\left(\cos \left(\phi_{0}\right)\right)^{2} \dot{\theta}_{0} \dot{L}_{1}-\rho_{1} \ddot{L}_{1} \cos \left(\phi_{1}\right) \sin \left(\theta_{1}\right) L_{0} \cos \left(\phi_{0}\right) \cos \left(\theta_{0}\right) L_{1}+\rho_{1} \ddot{L}_{1} \cos \left(\phi_{1}\right) \cos \left(\theta_{1}\right) L_{0} \cos \left(\phi_{0}\right) \sin \left(\theta_{0}\right) L_{1} \\
& -\rho_{2} \ddot{L}_{2} \cos \left(\phi_{2}\right) \sin \left(\theta_{2}\right) L_{0} \cos \left(\phi_{0}\right) \cos \left(\theta_{0}\right) L_{2}+\rho_{2} \ddot{L}_{2} \cos \left(\phi_{2}\right) \cos \left(\theta_{2}\right) L_{0} \cos \left(\phi_{0}\right) \sin \left(\theta_{0}\right) L_{2} \\
& +m_{1} \ddot{L}_{1} \cos \left(\phi_{1}\right) \cos \left(\theta_{1}\right) L_{0} \cos \left(\phi_{0}\right) \sin \left(\theta_{0}\right)-m_{1} \ddot{L}_{1} \cos \left(\phi_{1}\right) \sin \left(\theta_{1}\right) L_{0} \cos \left(\phi_{0}\right) \cos \left(\theta_{0}\right) \\
& +m_{2} \ddot{L}_{2} \cos \left(\phi_{2}\right) \cos \left(\theta_{2}\right) L_{0} \cos \left(\phi_{0}\right) \sin \left(\theta_{0}\right)-m_{2} \ddot{L}_{2} \cos \left(\phi_{2}\right) \sin \left(\theta_{2}\right) L_{0} \cos \left(\phi_{0}\right) \cos \left(\theta_{0}\right) \\
& +2 / 3 \rho_{0} \cos \left(\phi_{0}\right) \dot{\theta}_{0} L_{0}^{3} \sin \left(\phi_{0}\right) \dot{\phi}_{0}+1 / 2 \rho_{1} L_{1}^{2} \cos \left(\phi_{1}\right) \dot{\phi}_{1}^{2} \sin \left(\theta_{1}\right) L_{0} \cos \left(\phi_{0}\right) \cos \left(\theta_{0}\right) \\
& -m_{1} L_{1} \cos \left(\phi_{1}\right) \cos \left(\theta_{1}\right) \dot{\theta}_{1}^{2} L_{0} \cos \left(\phi_{0}\right) \sin \left(\theta_{0}\right)+\rho_{1} g L_{0} \cos \left(\phi_{0}\right) \sin \left(\theta_{0}\right) L_{1}
\end{aligned}
$$




$$
\begin{aligned}
& b_{2}=-2 m_{1} \dot{L}_{1} \sin \left(\phi_{1}\right) \dot{\phi}_{1} \sin \left(\theta_{1}\right) L_{0} \sin \left(\phi_{0}\right) \sin \left(\theta_{0}\right)+m_{1} \ddot{L}_{1} \cos \left(\phi_{1}\right) \cos \left(\theta_{1}\right) L_{0} \sin \left(\phi_{0}\right) \cos \left(\theta_{0}\right) \\
& -m_{2} L_{2} \cos \left(\phi_{2}\right) \dot{\phi}_{2}^{2} \cos \left(\theta_{2}\right) L_{0} \sin \left(\phi_{0}\right) \cos \left(\theta_{0}\right)-m_{2} \ddot{L}_{2} \sin \left(\phi_{2}\right) L_{0} \cos \left(\phi_{0}\right) \\
& -\rho_{2} \dot{L}_{2}^{2} \sin \left(\phi_{2}\right) L_{0} \cos \left(\phi_{0}\right)+m_{1} \ddot{L}_{1} \cos \left(\phi_{1}\right) \sin \left(\theta_{1}\right) L_{0} \sin \left(\phi_{0}\right) \sin \left(\theta_{0}\right) \\
& +2 \rho_{2} \dot{L}_{2} \cos \left(\phi_{2}\right) \cos \left(\theta_{2}\right) \dot{\theta}_{2} L_{0} \sin \left(\phi_{0}\right) \sin \left(\theta_{0}\right) L_{2}+\rho_{2} \dot{L}_{2}^{2} \cos \left(\phi_{2}\right) \sin \left(\theta_{2}\right) L_{0} \sin \left(\phi_{0}\right) \sin \left(\theta_{0}\right) \\
& -2 \rho_{1} L_{0} \dot{\phi}_{0} L_{1} \frac{d}{d t} L_{0}-2 m_{2} \dot{L}_{2} \sin \left(\phi_{2}\right) \dot{\phi}_{2} \cos \left(\theta_{2}\right) L_{0} \sin \left(\phi_{0}\right) \cos \left(\theta_{0}\right) \\
& -2 m_{2} \dot{L}_{2} \cos \left(\phi_{2}\right) \sin \left(\theta_{2}\right) \dot{\theta}_{2} L_{0} \sin \left(\phi_{0}\right) \cos \left(\theta_{0}\right)+2 m_{2} \dot{L}_{2} \cos \left(\phi_{2}\right) \cos \left(\theta_{2}\right) \dot{\theta}_{2} L_{0} \sin \left(\phi_{0}\right) \sin \left(\theta_{0}\right) \\
& -m_{2} L_{2} \cos \left(\phi_{2}\right) \sin \left(\theta_{2}\right) \dot{\theta}_{2}^{2} L_{0} \sin \left(\phi_{0}\right) \sin \left(\theta_{0}\right)-\rho_{2} \ddot{L}_{2} \sin \left(\phi_{2}\right) L_{0} \cos \left(\phi_{0}\right) L_{2} \\
& -2 \rho_{2} \dot{L}_{2} \cos \left(\phi_{2}\right) \dot{\phi}_{2} L_{0} \cos \left(\phi_{0}\right) L_{2}+m_{2} \ddot{L}_{2} \cos \left(\phi_{2}\right) \sin \left(\theta_{2}\right) L_{0} \sin \left(\phi_{0}\right) \sin \left(\theta_{0}\right) \\
& -\rho_{1} L_{0}^{2} \cos \left(\phi_{0}\right) \dot{\theta}_{0}^{2} L_{1} \sin \left(\phi_{0}\right)-1 / 2 \rho_{2} L_{2}^{2} \cos \left(\phi_{2}\right) \dot{\phi}_{2}^{2} \cos \left(\theta_{2}\right) L_{0} \sin \left(\phi_{0}\right) \cos \left(\theta_{0}\right) \\
& -2 m_{2} \dot{L}_{2} \sin \left(\phi_{2}\right) \dot{\phi}_{2} \sin \left(\theta_{2}\right) L_{0} \sin \left(\phi_{0}\right) \sin \left(\theta_{0}\right)+1 / 2 \rho_{2} L_{2}^{2} \sin \left(\phi_{2}\right) \dot{\phi}_{2}^{2} L_{0} \cos \left(\phi_{0}\right) \\
& -2 m_{2} L_{2} \sin \left(\phi_{2}\right) \dot{\phi}_{2} \cos \left(\theta_{2}\right) \dot{\theta}_{2} L_{0} \sin \left(\phi_{0}\right) \sin \left(\theta_{0}\right)-m_{1} L_{0}^{2} \cos \left(\phi_{0}\right) \dot{\theta}_{0}^{2} \sin \left(\phi_{0}\right) \\
& -2 m_{2} \dot{L}_{2} \cos \left(\phi_{2}\right) \dot{\phi}_{2} L_{0} \cos \left(\phi_{0}\right)-\rho_{1} \dot{L}_{1}^{2} \sin \left(\phi_{1}\right) L_{0} \cos \left(\phi_{0}\right) \\
& -\rho_{1} L_{1}^{2} \sin \left(\phi_{1}\right) \dot{\phi}_{1} \cos \left(\theta_{1}\right) \dot{\theta}_{1} L_{0} \sin \left(\phi_{0}\right) \sin \left(\theta_{0}\right)-1 / 2 \rho_{2} L_{2}{ }^{2} \cos \left(\phi_{2}\right) \dot{\phi}_{2}{ }^{2} \sin \left(\theta_{2}\right) L_{0} \sin \left(\phi_{0}\right) \sin \left(\theta_{0}\right) \\
& +m_{2} \ddot{L}_{2} \cos \left(\phi_{2}\right) \cos \left(\theta_{2}\right) L_{0} \sin \left(\phi_{0}\right) \cos \left(\theta_{0}\right)-\rho_{2} L_{2}^{2} \sin \left(\phi_{2}\right) \dot{\phi}_{2} \cos \left(\theta_{2}\right) \dot{\theta}_{2} L_{0} \sin \left(\phi_{0}\right) \sin \left(\theta_{0}\right) \\
& -1 / 2 \rho_{1} L_{1}^{2} \cos \left(\phi_{1}\right) \dot{\phi}_{1}^{2} \sin \left(\theta_{1}\right) L_{0} \sin \left(\phi_{0}\right) \sin \left(\theta_{0}\right)+\rho_{2} L_{2}^{2} \sin \left(\phi_{2}\right) \dot{\phi}_{2} \sin \left(\theta_{2}\right) \dot{\theta}_{2} L_{0} \sin \left(\phi_{0}\right) \cos \left(\theta_{0}\right) \\
& -\rho_{2} L_{0}^{2} \cos \left(\phi_{0}\right) \dot{\theta}_{0}^{2} L_{2} \sin \left(\phi_{0}\right)+m_{2} L_{2} \sin \left(\phi_{2}\right) \dot{\phi}_{2}^{2} L_{0} \cos \left(\phi_{0}\right)+\rho_{2} \ddot{L}_{2} \cos \left(\phi_{2}\right) \cos \left(\theta_{2}\right) L_{0} \sin \left(\phi_{0}\right) \cos \left(\theta_{0}\right) L_{2} \\
& -2 \rho_{2} \dot{L}_{2} \sin \left(\phi_{2}\right) \dot{\phi}_{2} \cos \left(\theta_{2}\right) L_{0} \sin \left(\phi_{0}\right) \cos \left(\theta_{0}\right) L_{2}+1 / 2 \rho_{1} L_{1}^{2} \sin \left(\phi_{1}\right) \dot{\phi}_{1}^{2} L_{0} \cos \left(\phi_{0}\right) \\
& -2 \rho_{1} \dot{L}_{1} \cos \left(\phi_{1}\right) \dot{\phi}_{1} L_{0} \cos \left(\phi_{0}\right) L_{1}-2 \rho_{2} \dot{L}_{2} \cos \left(\phi_{2}\right) \sin \left(\theta_{2}\right) \dot{\theta}_{2} L_{0} \sin \left(\phi_{0}\right) \cos \left(\theta_{0}\right) L_{2} \\
& +\rho_{2} \dot{L}_{2}^{2} \cos \left(\phi_{2}\right) \cos \left(\theta_{2}\right) L_{0} \sin \left(\phi_{0}\right) \cos \left(\theta_{0}\right)-1 / 2 \rho_{2} L_{2}^{2} \cos \left(\phi_{2}\right) \cos \left(\theta_{2}\right) \dot{\theta}_{2}^{2} L_{0} \sin \left(\phi_{0}\right) \cos \left(\theta_{0}\right) \\
& -1 / 2 \rho_{1} L_{1}^{2} \cos \left(\phi_{1}\right) \sin \left(\theta_{1}\right) \dot{\theta}_{1}^{2} L_{0} \sin \left(\phi_{0}\right) \sin \left(\theta_{0}\right)+\rho_{1} L_{1}^{2} \sin \left(\phi_{1}\right) \dot{\phi}_{1} \sin \left(\theta_{1}\right) \dot{\theta}_{1} L_{0} \sin \left(\phi_{0}\right) \cos \left(\theta_{0}\right) \\
& -1 / 2 \rho_{2} L_{2}^{2} \cos \left(\phi_{2}\right) \sin \left(\theta_{2}\right) \dot{\theta}_{2}^{2} L_{0} \sin \left(\phi_{0}\right) \sin \left(\theta_{0}\right)-2 \rho_{1} \dot{L}_{1} \sin \left(\phi_{1}\right) \dot{\phi}_{1} \sin \left(\theta_{1}\right) L_{0} \sin \left(\phi_{0}\right) \sin \left(\theta_{0}\right) L_{1} \\
& -1 / 2 \rho_{1} L_{1}^{2} \cos \left(\phi_{1}\right) \cos \left(\theta_{1}\right) \dot{\theta}_{1}^{2} L_{0} \sin \left(\phi_{0}\right) \cos \left(\theta_{0}\right)-m_{1} \ddot{L}_{1} \sin \left(\phi_{1}\right) L_{0} \cos \left(\phi_{0}\right) \\
& +\rho_{1} \dot{L}_{1}^{2} \cos \left(\phi_{1}\right) \sin \left(\theta_{1}\right) L_{0} \sin \left(\phi_{0}\right) \sin \left(\theta_{0}\right)-1 / 2 \rho_{1} L_{1}^{2} \cos \left(\phi_{1}\right) \dot{\phi}_{1}^{2} \cos \left(\theta_{1}\right) L_{0} \sin \left(\phi_{0}\right) \cos \left(\theta_{0}\right) \\
& -\rho_{1} \ddot{L}_{1} \sin \left(\phi_{1}\right) L_{0} \cos \left(\phi_{0}\right) L_{1}+2 \rho_{1} \dot{L}_{1} \cos \left(\phi_{1}\right) \cos \left(\theta_{1}\right) \dot{\theta}_{1} L_{0} \sin \left(\phi_{0}\right) \sin \left(\theta_{0}\right) L_{1} \\
& +2 m_{1} L_{1} \sin \left(\phi_{1}\right) \dot{\phi}_{1} \sin \left(\theta_{1}\right) \dot{\theta}_{1} L_{0} \sin \left(\phi_{0}\right) \cos \left(\theta_{0}\right)+Q_{2}-2 m_{1} \dot{L}_{1} \cos \left(\phi_{1}\right) \dot{\phi}_{1} L_{0} \cos \left(\phi_{0}\right) \\
& +\rho_{1} \ddot{L}_{1} \cos \left(\phi_{1}\right) \sin \left(\theta_{1}\right) L_{0} \sin \left(\phi_{0}\right) \sin \left(\theta_{0}\right) L_{1}-2 m_{1} L_{1} \sin \left(\phi_{1}\right) \dot{\phi}_{1} \cos \left(\theta_{1}\right) \dot{\theta}_{1} L_{0} \sin \left(\phi_{0}\right) \sin \left(\theta_{0}\right) \\
& +m_{1} L_{1} \sin \left(\phi_{1}\right) \dot{\phi}_{1}^{2} L_{0} \cos \left(\phi_{0}\right)+\rho_{1} \ddot{L}_{1} \cos \left(\phi_{1}\right) \cos \left(\theta_{1}\right) L_{0} \sin \left(\phi_{0}\right) \cos \left(\theta_{0}\right) L_{1}-m_{2} L_{0}{ }^{2} \cos \left(\phi_{0}\right) \dot{\theta}_{0}^{2} \sin \left(\phi_{0}\right) \\
& -2 \rho_{1} \dot{L}_{1} \sin \left(\phi_{1}\right) \dot{\phi}_{1} \cos \left(\theta_{1}\right) L_{0} \sin \left(\phi_{0}\right) \cos \left(\theta_{0}\right) L_{1}-2 \rho_{1} \dot{L}_{1} \cos \left(\phi_{1}\right) \sin \left(\theta_{1}\right) \dot{\theta}_{1} L_{0} \sin \left(\phi_{0}\right) \cos \left(\theta_{0}\right) L_{1} \\
& +\rho_{1} \dot{L}_{1}^{2} \cos \left(\phi_{1}\right) \cos \left(\theta_{1}\right) L_{0} \sin \left(\phi_{0}\right) \cos \left(\theta_{0}\right)+2 m_{2} L_{2} \sin \left(\phi_{2}\right) \dot{\phi}_{2} \sin \left(\theta_{2}\right) \dot{\theta}_{2} L_{0} \sin \left(\phi_{0}\right) \cos \left(\theta_{0}\right) \\
& +2 m_{1} \dot{L}_{1} \cos \left(\phi_{1}\right) \cos \left(\theta_{1}\right) \dot{\theta}_{1} L_{0} \sin \left(\phi_{0}\right) \sin \left(\theta_{0}\right)+\rho_{2} \ddot{L}_{2} \cos \left(\phi_{2}\right) \sin \left(\theta_{2}\right) L_{0} \sin \left(\phi_{0}\right) \sin \left(\theta_{0}\right) L_{2} \\
& -m_{2} L_{2} \cos \left(\phi_{2}\right) \dot{\phi}_{2}^{2} \sin \left(\theta_{2}\right) L_{0} \sin \left(\phi_{0}\right) \sin \left(\theta_{0}\right)-m_{2} L_{2} \cos \left(\phi_{2}\right) \cos \left(\theta_{2}\right) \dot{\theta}_{2}^{2} L_{0} \sin \left(\phi_{0}\right) \cos \left(\theta_{0}\right) \\
& -2 \rho_{2} L_{0} \dot{\phi}_{0} L_{2} \dot{L}_{0}-2 \rho_{2} \dot{L}_{2} \sin \left(\phi_{2}\right) \dot{\phi}_{2} \sin \left(\theta_{2}\right) L_{0} \sin \left(\phi_{0}\right) \sin \left(\theta_{0}\right) L_{2} \\
& -2 m_{1} \dot{L}_{1} \sin \left(\phi_{1}\right) \dot{\phi}_{1} \cos \left(\theta_{1}\right) L_{0} \sin \left(\phi_{0}\right) \cos \left(\theta_{0}\right)-m_{1} L_{1} \cos \left(\phi_{1}\right) \cos \left(\theta_{1}\right) \dot{\theta}_{1}^{2} L_{0} \sin \left(\phi_{0}\right) \cos \left(\theta_{0}\right) \\
& +\rho_{1} g L_{0} \sin \left(\phi_{0}\right) \cos \left(\theta_{0}\right) L_{1}+1 / 2 \rho_{0} g \sin \left(\phi_{0}\right) \cos \left(\theta_{0}\right) L_{0}{ }^{2}+m_{1} L_{0} \sin \left(\phi_{0}\right) \cos \left(\theta_{0}\right)+m_{2} L_{0} \sin \left(\phi_{0}\right) \cos \left(\theta_{0}\right) \\
& -\rho_{1} L_{0}^{2} \dot{\phi}_{0} \dot{L}_{1}-\rho_{2} L_{0}^{2} \dot{\phi}_{0} \dot{L}_{2}-\rho_{0} \dot{\phi}_{0} L_{0}^{2} \dot{L}_{0}-2 m_{1} L_{0} \dot{\phi}_{0} \dot{L}_{0}-2 m_{2} L_{0} \dot{\phi}_{0} \dot{L}_{0}+\rho_{2} g L_{0} \sin \left(\phi_{0}\right) \cos \left(\theta_{0}\right) L_{2} \\
& -m_{1} L_{1} \cos \left(\phi_{1}\right) \sin \left(\theta_{1}\right) \dot{\theta}_{1}^{2} L_{0} \sin \left(\phi_{0}\right) \sin \left(\theta_{0}\right)-m_{1} L_{1} \cos \left(\phi_{1}\right) \dot{\phi}_{1}^{2} \cos \left(\theta_{1}\right) L_{0} \sin \left(\phi_{0}\right) \cos \left(\theta_{0}\right) \\
& -1 / 3 \rho_{0} \cos \left(\phi_{0}\right) \dot{\theta}_{0}{ }^{2} \sin \left(\phi_{0}\right) L_{0}{ }^{3}-2 m_{1} \dot{L}_{1} \cos \left(\phi_{1}\right) \sin \left(\theta_{1}\right) \dot{\theta}_{1} L_{0} \sin \left(\phi_{0}\right) \cos \left(\theta_{0}\right) \\
& -m_{1} L_{1} \cos \left(\phi_{1}\right) \dot{\phi}_{1}^{2} \sin \left(\theta_{1}\right) L_{0} \sin \left(\phi_{0}\right) \sin \left(\theta_{0}\right)
\end{aligned}
$$




$$
\begin{aligned}
& b_{3}=m_{1} L_{0} \dot{\phi}_{0}^{2}+m_{2} L_{0} \dot{\phi}_{0}^{2}-2 m_{1} \dot{L}_{1} \cos \left(\phi_{1}\right) \dot{\phi}_{1} \sin \left(\phi_{0}\right)+1 / 2 \rho_{1} L_{1}^{2} \cos \left(\phi_{1}\right) \dot{\phi}_{1}^{2} \sin \left(\theta_{1}\right) \cos \left(\phi_{0}\right) \sin \left(\theta_{0}\right) \\
& +1 / 2 \rho_{1} L_{1}^{2} \cos \left(\phi_{1}\right) \sin \left(\theta_{1}\right) \dot{\theta}_{1}^{2} \cos \left(\phi_{0}\right) \sin \left(\theta_{0}\right)+1 / 2 \rho_{1} L_{1}^{2} \cos \left(\phi_{1}\right) \dot{\phi}_{1}^{2} \cos \left(\theta_{1}\right) \cos \left(\phi_{0}\right) \cos \left(\theta_{0}\right) \\
& -\rho_{1} L_{1}^{2} \sin \left(\phi_{1}\right) \dot{\phi}_{1} \sin \left(\theta_{1}\right) \dot{\theta}_{1} \cos \left(\phi_{0}\right) \cos \left(\theta_{0}\right)+1 / 2 \rho_{1} L_{1}^{2} \cos \left(\phi_{1}\right) \cos \left(\theta_{1}\right) \dot{\theta}_{1}^{2} \cos \left(\phi_{0}\right) \cos \left(\theta_{0}\right) \\
& -2 \rho_{1} \dot{L}_{1} \cos \left(\phi_{1}\right) \dot{\phi}_{1} \sin \left(\phi_{0}\right) L_{1}-\rho_{1} \ddot{L}_{1} \cos \left(\phi_{1}\right) \sin \left(\theta_{1}\right) \cos \left(\phi_{0}\right) \sin \left(\theta_{0}\right) L_{1} \\
& +2 \rho_{1} \dot{L}_{1} \sin \left(\phi_{1}\right) \dot{\phi}_{1} \sin \left(\theta_{1}\right) \cos \left(\phi_{0}\right) \sin \left(\theta_{0}\right) L_{1}-2 \rho_{1} \dot{L}_{1} \cos \left(\phi_{1}\right) \cos \left(\theta_{1}\right) \dot{\theta}_{1} \cos \left(\phi_{0}\right) \sin \left(\theta_{0}\right) L_{1} \\
& -\rho_{1} \dot{L}_{1}^{2} \cos \left(\phi_{1}\right) \sin \left(\theta_{1}\right) \cos \left(\phi_{0}\right) \sin \left(\theta_{0}\right)-m_{1} \ddot{L}_{0}-1 / 2 \rho_{0} \dot{L}_{0}{ }^{2}-m_{1} \cos \left(\phi_{0}\right) \cos \left(\theta_{0}\right) \\
& +1 / 2 \rho_{0} L_{0}^{2}\left(\cos \left(\phi_{0}\right)\right)^{2} \dot{\theta}_{0}^{2}+m_{1} L_{0}\left(\cos \left(\phi_{0}\right)\right)^{2} \dot{\theta}_{0}^{2}+m_{2} L_{0}\left(\cos \left(\phi_{0}\right)\right)^{2} \dot{\theta}_{0}^{2}-\rho_{2} \dot{L}_{2}^{2} \sin \left(\phi_{2}\right) \sin \left(\phi_{0}\right) \\
& -\rho_{2} \ddot{L}_{0} L_{2}+1 / 2 \rho_{0} L_{0}^{2} \dot{\phi}_{0}^{2}-\rho_{0} \ddot{L}_{0} L_{0}+Q_{3}-\rho_{1} \ddot{L}_{0} L_{1}-2 \rho_{2} \dot{L}_{2} \cos \left(\phi_{2}\right) \dot{\phi}_{2} \sin \left(\phi_{0}\right) L_{2} \\
& -m_{2} \cos \left(\phi_{0}\right) \cos \left(\theta_{0}\right)-\rho_{1} \dot{L}_{0} \dot{L}_{1}-\rho_{0} g \cos \left(\phi_{0}\right) \cos \left(\theta_{0}\right) L_{0}-\rho_{1} g \cos \left(\phi_{0}\right) \cos \left(\theta_{0}\right) L_{1}-\rho_{2} g \cos \left(\phi_{0}\right) \cos \left(\theta_{0}\right) L_{2} \\
& +1 / 2 \rho_{2} L_{2}^{2} \cos \left(\phi_{2}\right) \sin \left(\theta_{2}\right) \dot{\theta}_{2}^{2} \cos \left(\phi_{0}\right) \sin \left(\theta_{0}\right)-\rho_{2} L_{2}^{2} \sin \left(\phi_{2}\right) \dot{\phi}_{2} \sin \left(\theta_{2}\right) \dot{\theta}_{2} \cos \left(\phi_{0}\right) \cos \left(\theta_{0}\right) \\
& -m_{2} \ddot{L}_{0}+1 / 2 \rho_{2} L_{2}{ }^{2} \cos \left(\phi_{2}\right) \cos \left(\theta_{2}\right) \dot{\theta}_{2}{ }^{2} \cos \left(\phi_{0}\right) \cos \left(\theta_{0}\right)+1 / 2 \rho_{2} L_{2}{ }^{2} \sin \left(\phi_{2}\right) \dot{\phi}_{2}{ }^{2} \sin \left(\phi_{0}\right) \\
& -\rho_{2} \ddot{L}_{2} \cos \left(\phi_{2}\right) \sin \left(\theta_{2}\right) \cos \left(\phi_{0}\right) \sin \left(\theta_{0}\right) L_{2}-\rho_{2} \dot{L}_{2}^{2} \cos \left(\phi_{2}\right) \sin \left(\theta_{2}\right) \cos \left(\phi_{0}\right) \sin \left(\theta_{0}\right) \\
& -\rho_{1} \ddot{L}_{1} \cos \left(\phi_{1}\right) \cos \left(\theta_{1}\right) \cos \left(\phi_{0}\right) \cos \left(\theta_{0}\right) L_{1}+2 \rho_{1} \dot{L}_{1} \sin \left(\phi_{1}\right) \dot{\phi}_{1} \cos \left(\theta_{1}\right) \cos \left(\phi_{0}\right) \cos \left(\theta_{0}\right) L_{1} \\
& +2 \rho_{1} \dot{L}_{1} \cos \left(\phi_{1}\right) \sin \left(\theta_{1}\right) \dot{\theta}_{1} \cos \left(\phi_{0}\right) \cos \left(\theta_{0}\right) L_{1}-\rho_{1} \dot{L}_{1}^{2} \cos \left(\phi_{1}\right) \cos \left(\theta_{1}\right) \cos \left(\phi_{0}\right) \cos \left(\theta_{0}\right) \\
& +1 / 2 \rho_{1} L_{1}^{2} \sin \left(\phi_{1}\right) \dot{\phi}_{1}^{2} \sin \left(\phi_{0}\right)+\rho_{2} L_{0}\left(\cos \left(\phi_{0}\right)\right)^{2} \dot{\theta}_{0}^{2} L_{2}-2 m_{2} L_{2} \sin \left(\phi_{2}\right) \dot{\phi}_{2} \sin \left(\theta_{2}\right) \dot{\theta}_{2} \cos \left(\phi_{0}\right) \cos \left(\theta_{0}\right) \\
& +2 m_{2} \dot{L}_{2} \sin \left(\phi_{2}\right) \dot{\phi}_{2} \cos \left(\theta_{2}\right) \cos \left(\phi_{0}\right) \cos \left(\theta_{0}\right)+m_{2} L_{2} \cos \left(\phi_{2}\right) \dot{\phi}_{2}^{2} \cos \left(\theta_{2}\right) \cos \left(\phi_{0}\right) \cos \left(\theta_{0}\right) \\
& +m_{2} L_{2} \cos \left(\phi_{2}\right) \cos \left(\theta_{2}\right) \dot{\theta}_{2}^{2} \cos \left(\phi_{0}\right) \cos \left(\theta_{0}\right)+2 m_{2} \dot{L}_{2} \cos \left(\phi_{2}\right) \sin \left(\theta_{2}\right) \dot{\theta}_{2} \cos \left(\phi_{0}\right) \cos \left(\theta_{0}\right) \\
& +m_{2} L_{2} \cos \left(\phi_{2}\right) \dot{\phi}_{2}^{2} \sin \left(\theta_{2}\right) \cos \left(\phi_{0}\right) \sin \left(\theta_{0}\right)+m_{2} L_{2} \cos \left(\phi_{2}\right) \sin \left(\theta_{2}\right) \dot{\theta}_{2}^{2} \cos \left(\phi_{0}\right) \sin \left(\theta_{0}\right) \\
& +2 m_{2} L_{2} \sin \left(\phi_{2}\right) \dot{\phi}_{2} \cos \left(\theta_{2}\right) \dot{\theta}_{2} \cos \left(\phi_{0}\right) \sin \left(\theta_{0}\right)-2 m_{2} \dot{L}_{2} \cos \left(\phi_{2}\right) \cos \left(\theta_{2}\right) \dot{\theta}_{2} \cos \left(\phi_{0}\right) \sin \left(\theta_{0}\right) \\
& +2 m_{2} \dot{L}_{2} \sin \left(\phi_{2}\right) \dot{\phi}_{2} \sin \left(\theta_{2}\right) \cos \left(\phi_{0}\right) \sin \left(\theta_{0}\right)+1 / 2 \rho_{2} L_{2}^{2} \cos \left(\phi_{2}\right) \dot{\phi}_{2}^{2} \sin \left(\theta_{2}\right) \cos \left(\phi_{0}\right) \sin \left(\theta_{0}\right) \\
& +\rho_{2} L_{2}^{2} \sin \left(\phi_{2}\right) \dot{\phi}_{2} \cos \left(\theta_{2}\right) \dot{\theta}_{2} \cos \left(\phi_{0}\right) \sin \left(\theta_{0}\right)+1 / 2 \rho_{2} L_{2}{ }^{2} \cos \left(\phi_{2}\right) \dot{\phi}_{2}^{2} \cos \left(\theta_{2}\right) \cos \left(\phi_{0}\right) \cos \left(\theta_{0}\right) \\
& -\rho_{2} \ddot{L}_{2} \sin \left(\phi_{2}\right) \sin \left(\phi_{0}\right) L_{2}+2 \rho_{2} \dot{L}_{2} \sin \left(\phi_{2}\right) \dot{\phi}_{2} \sin \left(\theta_{2}\right) \cos \left(\phi_{0}\right) \sin \left(\theta_{0}\right) L_{2} \\
& -2 \rho_{2} \dot{L}_{2} \cos \left(\phi_{2}\right) \cos \left(\theta_{2}\right) \dot{\theta}_{2} \cos \left(\phi_{0}\right) \sin \left(\theta_{0}\right) L_{2}-\rho_{2} \ddot{L}_{2} \cos \left(\phi_{2}\right) \cos \left(\theta_{2}\right) \cos \left(\phi_{0}\right) \cos \left(\theta_{0}\right) L_{2} \\
& +2 \rho_{2} \dot{L}_{2} \sin \left(\phi_{2}\right) \dot{\phi}_{2} \cos \left(\theta_{2}\right) \cos \left(\phi_{0}\right) \cos \left(\theta_{0}\right) L_{2}+2 \rho_{2} \dot{L}_{2} \cos \left(\phi_{2}\right) \sin \left(\theta_{2}\right) \dot{\theta}_{2} \cos \left(\phi_{0}\right) \cos \left(\theta_{0}\right) L_{2} \\
& -\rho_{2} \dot{L}_{2}^{2} \cos \left(\phi_{2}\right) \cos \left(\theta_{2}\right) \cos \left(\phi_{0}\right) \cos \left(\theta_{0}\right)-m_{2} \ddot{L}_{2} \cos \left(\phi_{2}\right) \cos \left(\theta_{2}\right) \cos \left(\phi_{0}\right) \cos \left(\theta_{0}\right) \\
& -m_{2} \ddot{L}_{2} \cos \left(\phi_{2}\right) \sin \left(\theta_{2}\right) \cos \left(\phi_{0}\right) \sin \left(\theta_{0}\right)-2 m_{2} \dot{L}_{2} \cos \left(\phi_{2}\right) \dot{\phi}_{2} \sin \left(\phi_{0}\right)-\rho_{2} \dot{L}_{0} \dot{L}_{2} \\
& +\rho_{1} L_{0} \dot{\phi}_{0}^{2} L_{1}+\rho_{2} L_{0} \dot{\phi}_{0}^{2} L_{2}-\rho_{1} \dot{L}_{1}^{2} \sin \left(\phi_{1}\right) \sin \left(\phi_{0}\right)-m_{1} \ddot{L}_{1} \sin \left(\phi_{1}\right) \sin \left(\phi_{0}\right) \\
& -m_{2} \ddot{L}_{2} \sin \left(\phi_{2}\right) \sin \left(\phi_{0}\right)+m_{2} L_{2} \sin \left(\phi_{2}\right) \dot{\phi}_{2}^{2} \sin \left(\phi_{0}\right)+\rho_{1} L_{0}\left(\cos \left(\phi_{0}\right)\right)^{2} \dot{\theta}_{0}^{2} L_{1} \\
& +m_{1} L_{1} \cos \left(\phi_{1}\right) \sin \left(\theta_{1}\right) \dot{\theta}_{1}^{2} \cos \left(\phi_{0}\right) \sin \left(\theta_{0}\right)+m_{1} L_{1} \cos \left(\phi_{1}\right) \dot{\phi}_{1}^{2} \sin \left(\theta_{1}\right) \cos \left(\phi_{0}\right) \sin \left(\theta_{0}\right) \\
& +2 m_{1} L_{1} \sin \left(\phi_{1}\right) \dot{\phi}_{1} \cos \left(\theta_{1}\right) \dot{\theta}_{1} \cos \left(\phi_{0}\right) \sin \left(\theta_{0}\right)-m_{1} \ddot{L}_{1} \cos \left(\phi_{1}\right) \sin \left(\theta_{1}\right) \cos \left(\phi_{0}\right) \sin \left(\theta_{0}\right) \\
& +m_{1} L_{1} \sin \left(\phi_{1}\right) \dot{\phi}_{1}^{2} \sin \left(\phi_{0}\right)-\rho_{1} \ddot{L}_{1} \sin \left(\phi_{1}\right) \sin \left(\phi_{0}\right) L_{1}+\rho_{1} L_{1}^{2} \sin \left(\phi_{1}\right) \dot{\phi}_{1} \cos \left(\theta_{1}\right) \dot{\theta}_{1} \cos \left(\phi_{0}\right) \sin \left(\theta_{0}\right) \\
& +2 m_{1} \dot{L}_{1} \sin \left(\phi_{1}\right) \dot{\phi}_{1} \sin \left(\theta_{1}\right) \cos \left(\phi_{0}\right) \sin \left(\theta_{0}\right)-2 m_{1} \dot{L}_{1} \cos \left(\phi_{1}\right) \cos \left(\theta_{1}\right) \dot{\theta}_{1} \cos \left(\phi_{0}\right) \sin \left(\theta_{0}\right) \\
& +2 m_{1} \dot{L}_{1} \cos \left(\phi_{1}\right) \sin \left(\theta_{1}\right) \dot{\theta}_{1} \cos \left(\phi_{0}\right) \cos \left(\theta_{0}\right)-2 m_{1} L_{1} \sin \left(\phi_{1}\right) \dot{\phi}_{1} \sin \left(\theta_{1}\right) \dot{\theta}_{1} \cos \left(\phi_{0}\right) \cos \left(\theta_{0}\right) \\
& +m_{1} L_{1} \cos \left(\phi_{1}\right) \cos \left(\theta_{1}\right) \dot{\theta}_{1}^{2} \cos \left(\phi_{0}\right) \cos \left(\theta_{0}\right)+2 m_{1} \dot{L}_{1} \sin \left(\phi_{1}\right) \dot{\phi}_{1} \cos \left(\theta_{1}\right) \cos \left(\phi_{0}\right) \cos \left(\theta_{0}\right) \\
& -m_{1} \ddot{L}_{1} \cos \left(\phi_{1}\right) \cos \left(\theta_{1}\right) \cos \left(\phi_{0}\right) \cos \left(\theta_{0}\right)+m_{1} L_{1} \cos \left(\phi_{1}\right) \dot{\phi}_{1}^{2} \cos \left(\theta_{1}\right) \cos \left(\phi_{0}\right) \cos \left(\theta_{0}\right)
\end{aligned}
$$




$$
\begin{aligned}
& b_{4}=m_{1} L_{1} \cos \left(\phi_{1}\right) \sin \left(\theta_{1}\right)-\rho_{1} L_{1}^{2} \cos \left(\phi_{1}\right) \sin \left(\theta_{1}\right) \dot{L}_{0} \cos \left(\phi_{0}\right) \sin \left(\theta_{0}\right) \dot{\theta}_{0} \\
& -2 m_{1} L_{1}\left(\cos \left(\phi_{1}\right)\right)^{2} \dot{\theta}_{1} \dot{L}_{1}+Q_{4}+2 m_{1} L_{1}^{2} \cos \left(\phi_{1}\right) \dot{\theta}_{1} \sin \left(\phi_{1}\right) \dot{\phi}_{1}+1 / 2 \rho_{1} g \cos \left(\phi_{1}\right) \sin \left(\theta_{1}\right) L_{1}^{2} \\
& +2 / 3 \rho_{1} \cos \left(\phi_{1}\right) \dot{\theta}_{1} L_{1}^{3} \sin \left(\phi_{1}\right) \dot{\phi}_{1}-1 / 2 \rho_{1} L_{1}^{2} \cos \left(\phi_{1}\right) \sin \left(\theta_{1}\right) L_{0} \cos \left(\phi_{0}\right) \cos \left(\theta_{0}\right) \dot{\theta}_{0}^{2} \\
& -\rho_{1} L_{1}^{2} \cos \left(\phi_{1}\right) \sin \left(\theta_{1}\right) \dot{L}_{0} \sin \left(\phi_{0}\right) \dot{\phi}_{0} \cos \left(\theta_{0}\right)-1 / 2 \rho_{1} L_{1}^{2} \cos \left(\phi_{1}\right) \sin \left(\theta_{1}\right) L_{0} \cos \left(\phi_{0}\right) \dot{\phi}_{0}^{2} \cos \left(\theta_{0}\right) \\
& +1 / 2 \rho_{1} L_{1}^{2} \cos \left(\phi_{1}\right) \cos \left(\theta_{1}\right) L_{0} \cos \left(\phi_{0}\right) \sin \left(\theta_{0}\right) \dot{\theta}_{0}^{2}+1 / 2 \rho_{1} L_{1}^{2} \cos \left(\phi_{1}\right) \sin \left(\theta_{1}\right) \ddot{L}_{0} \cos \left(\phi_{0}\right) \cos \left(\theta_{0}\right) \\
& +\rho_{1} L_{1}^{2} \cos \left(\phi_{1}\right) \cos \left(\theta_{1}\right) \dot{L}_{0} \sin \left(\phi_{0}\right) \dot{\phi}_{0} \sin \left(\theta_{0}\right)-\rho_{1} L_{1}^{2} \cos \left(\phi_{1}\right) \cos \left(\theta_{1}\right) \dot{L}_{0} \cos \left(\phi_{0}\right) \cos \left(\theta_{0}\right) \dot{\theta}_{0} \\
& -1 / 2 \rho_{1} L_{1}^{2} \cos \left(\phi_{1}\right) \cos \left(\theta_{1}\right) \ddot{L}_{0} \cos \left(\phi_{0}\right) \sin \left(\theta_{0}\right)-\rho_{1}\left(\cos \left(\phi_{1}\right)\right)^{2} \dot{\theta}_{1} L_{1}^{2} \dot{L}_{1} \\
& +\rho_{1} L_{1}^{2} \cos \left(\phi_{1}\right) \sin \left(\theta_{1}\right) L_{0} \sin \left(\phi_{0}\right) \dot{\phi}_{0} \sin \left(\theta_{0}\right) \dot{\theta}_{0}+1 / 2 \rho_{1} L_{1}^{2} \cos \left(\phi_{1}\right) \cos \left(\theta_{1}\right) L_{0} \cos \left(\phi_{0}\right) \dot{\phi}_{0}{ }^{2} \sin \left(\theta_{0}\right) \\
& +m_{1} L_{1} \cos \left(\phi_{1}\right) \sin \left(\theta_{1}\right) \ddot{L}_{0} \cos \left(\phi_{0}\right) \cos \left(\theta_{0}\right)-m_{1} L_{1} \cos \left(\phi_{1}\right) \sin \left(\theta_{1}\right) L_{0} \cos \left(\phi_{0}\right) \cos \left(\theta_{0}\right) \dot{\theta}_{0}^{2} \\
& +2 m_{1} L_{1} \cos \left(\phi_{1}\right) \sin \left(\theta_{1}\right) L_{0} \sin \left(\phi_{0}\right) \dot{\phi}_{0} \sin \left(\theta_{0}\right) \dot{\theta}_{0}-2 m_{1} L_{1} \cos \left(\phi_{1}\right) \sin \left(\theta_{1}\right) \dot{L}_{0} \cos \left(\phi_{0}\right) \sin \left(\theta_{0}\right) \dot{\theta}_{0} \\
& +m_{1} L_{1} \cos \left(\phi_{1}\right) \cos \left(\theta_{1}\right) L_{0} \cos \left(\phi_{0}\right) \sin \left(\theta_{0}\right) \dot{\theta}_{0}^{2}+2 m_{1} L_{1} \cos \left(\phi_{1}\right) \cos \left(\theta_{1}\right) \dot{L}_{0} \sin \left(\phi_{0}\right) \dot{\phi}_{0} \sin \left(\theta_{0}\right) \\
& +2 m_{1} L_{1} \cos \left(\phi_{1}\right) \cos \left(\theta_{1}\right) L_{0} \sin \left(\phi_{0}\right) \dot{\phi}_{0} \cos \left(\theta_{0}\right) \dot{\theta}_{0}-m_{1} L_{1} \cos \left(\phi_{1}\right) \cos \left(\theta_{1}\right) \ddot{L}_{0} \cos \left(\phi_{0}\right) \sin \left(\theta_{0}\right) \\
& -2 m_{1} L_{1} \cos \left(\phi_{1}\right) \cos \left(\theta_{1}\right) \dot{L}_{0} \cos \left(\phi_{0}\right) \cos \left(\theta_{0}\right) \dot{\theta}_{0}+m_{1} L_{1} \cos \left(\phi_{1}\right) \cos \left(\theta_{1}\right) L_{0} \cos \left(\phi_{0}\right) \dot{\phi}_{0}^{2} \sin \left(\theta_{0}\right) \\
& -2 m_{1} L_{1} \cos \left(\phi_{1}\right) \sin \left(\theta_{1}\right) \dot{L}_{0} \sin \left(\phi_{0}\right) \dot{\phi}_{0} \cos \left(\theta_{0}\right)-m_{1} L_{1} \cos \left(\phi_{1}\right) \sin \left(\theta_{1}\right) L_{0} \cos \left(\phi_{0}\right) \dot{\phi}_{0}^{2} \cos \left(\theta_{0}\right) \\
& +\rho_{1} L_{1}^{2} \cos \left(\phi_{1}\right) \cos \left(\theta_{1}\right) L_{0} \sin \left(\phi_{0}\right) \dot{\phi}_{0} \cos \left(\theta_{0}\right) \dot{\theta}_{0}
\end{aligned}
$$

$$
\begin{aligned}
& b_{5}=Q_{5}-2 m_{1} L_{1} \cos \left(\phi_{1}\right) \dot{L}_{0} \cos \left(\phi_{0}\right) \dot{\phi}_{0}+m_{1} L_{1} \sin \left(\phi_{1}\right) \sin \left(\theta_{1}\right) \ddot{L}_{0} \cos \left(\phi_{0}\right) \sin \left(\theta_{0}\right) \\
& -2 m_{1} L_{1} \sin \left(\phi_{1}\right) \sin \left(\theta_{1}\right) \dot{L}_{0} \sin \left(\phi_{0}\right) \dot{\phi}_{0} \sin \left(\theta_{0}\right)-1 / 3 \rho_{1} \cos \left(\phi_{1}\right) \dot{\theta}_{1}^{2} \sin \left(\phi_{1}\right) L_{1}^{3} \\
& -1 / 2 \rho_{1} L_{1}^{2} \cos \left(\phi_{1}\right) \ddot{L}_{0} \sin \left(\phi_{0}\right)-\rho_{1} L_{1}^{2} \sin \left(\phi_{1}\right) \sin \left(\theta_{1}\right) L_{0} \sin \left(\phi_{0}\right) \dot{\phi}_{0} \cos \left(\theta_{0}\right) \dot{\theta}_{0} \\
& +m_{1} L_{1} \cos \left(\phi_{1}\right) L_{0} \sin \left(\phi_{0}\right) \dot{\phi}_{0}^{2}-2 m_{1} L_{1} \sin \left(\phi_{1}\right) \cos \left(\theta_{1}\right) \dot{L}_{0} \cos \left(\phi_{0}\right) \sin \left(\theta_{0}\right) \dot{\theta}_{0} \\
& -m_{1} L_{1} \sin \left(\phi_{1}\right) \cos \left(\theta_{1}\right) L_{0} \cos \left(\phi_{0}\right) \cos \left(\theta_{0}\right) \dot{\theta}_{0}^{2}-m_{1} L_{1} \sin \left(\phi_{1}\right) \sin \left(\theta_{1}\right) L_{0} \cos \left(\phi_{0}\right) \dot{\phi}_{0}^{2} \sin \left(\theta_{0}\right) \\
& -2 m_{1} L_{1} \sin \left(\phi_{1}\right) \sin \left(\theta_{1}\right) L_{0} \sin \left(\phi_{0}\right) \dot{\phi}_{0} \cos \left(\theta_{0}\right) \dot{\theta}_{0}-m_{1} L_{1} \cos \left(\phi_{1}\right) \ddot{L}_{0} \sin \left(\phi_{0}\right) \\
& +2 m_{1} L_{1} \sin \left(\phi_{1}\right) \cos \left(\theta_{1}\right) L_{0} \sin \left(\phi_{0}\right) \dot{\phi}_{0} \sin \left(\theta_{0}\right) \dot{\theta}_{0}-2 m_{1} L_{1} \sin \left(\phi_{1}\right) \cos \left(\theta_{1}\right) \dot{L}_{0} \sin \left(\phi_{0}\right) \dot{\phi}_{0} \cos \left(\theta_{0}\right) \\
& +m_{1} L_{1} \sin \left(\phi_{1}\right) \cos \left(\theta_{1}\right) \ddot{L}_{0} \cos \left(\phi_{0}\right) \cos \left(\theta_{0}\right)-m_{1} L_{1} \sin \left(\phi_{1}\right) \sin \left(\theta_{1}\right) L_{0} \cos \left(\phi_{0}\right) \sin \left(\theta_{0}\right) \dot{\theta}_{0}^{2} \\
& +2 m_{1} L_{1} \sin \left(\phi_{1}\right) \sin \left(\theta_{1}\right) \dot{L}_{0} \cos \left(\phi_{0}\right) \cos \left(\theta_{0}\right) \dot{\theta}_{0}-m_{1} L_{1} \sin \left(\phi_{1}\right) \cos \left(\theta_{1}\right) L_{0} \cos \left(\phi_{0}\right) \dot{\phi}_{0}^{2} \cos \left(\theta_{0}\right) \\
& -m_{1} L_{1}^{2} \cos \left(\phi_{1}\right) \dot{\theta}_{1}^{2} \sin \left(\phi_{1}\right)+1 / 2 \rho_{1} g \sin \left(\phi_{1}\right) \cos \left(\theta_{1}\right) L_{1}^{2}+1 / 2 \rho_{1} L_{1}^{2} \sin \left(\phi_{1}\right) \cos \left(\theta_{1}\right) \ddot{L}_{0} \cos \left(\phi_{0}\right) \cos \left(\theta_{0}\right) \\
& +1 / 2 \rho_{1} L_{1}^{2} \sin \left(\phi_{1}\right) \sin \left(\theta_{1}\right) \ddot{L}_{0} \cos \left(\phi_{0}\right) \sin \left(\theta_{0}\right)-\rho_{1} L_{1}^{2} \cos \left(\phi_{1}\right) \dot{L}_{0} \cos \left(\phi_{0}\right) \dot{\phi}_{0}+1 / 2 \rho_{1} L_{1}^{2} \cos \left(\phi_{1}\right) L_{0} \sin \left(\phi_{0}\right) \dot{\phi}_{0}{ }^{2} \\
& -1 / 2 \rho_{1} L_{1}^{2} \sin \left(\phi_{1}\right) \sin \left(\theta_{1}\right) L_{0} \cos \left(\phi_{0}\right) \sin \left(\theta_{0}\right) \dot{\theta}_{0}^{2}-\rho_{1} L_{1}^{2} \sin \left(\phi_{1}\right) \cos \left(\theta_{1}\right) \dot{L}_{0} \cos \left(\phi_{0}\right) \sin \left(\theta_{0}\right) \dot{\theta}_{0} \\
& -1 / 2 \rho_{1} L_{1}^{2} \sin \left(\phi_{1}\right) \sin \left(\theta_{1}\right) L_{0} \cos \left(\phi_{0}\right) \dot{\phi}_{0}^{2} \sin \left(\theta_{0}\right)-1 / 2 \rho_{1} L_{1}^{2} \sin \left(\phi_{1}\right) \cos \left(\theta_{1}\right) L_{0} \cos \left(\phi_{0}\right) \cos \left(\theta_{0}\right) \dot{\theta}_{0}^{2} \\
& -1 / 2 \rho_{1} L_{1}^{2} \sin \left(\phi_{1}\right) \cos \left(\theta_{1}\right) L_{0} \cos \left(\phi_{0}\right) \dot{\phi}_{0}^{2} \cos \left(\theta_{0}\right)+\rho_{1} L_{1}^{2} \sin \left(\phi_{1}\right) \cos \left(\theta_{1}\right) L_{0} \sin \left(\phi_{0}\right) \dot{\phi}_{0} \sin \left(\theta_{0}\right) \dot{\theta}_{0} \\
& -\rho_{1} L_{1}^{2} \sin \left(\phi_{1}\right) \sin \left(\theta_{1}\right) \dot{L}_{0} \sin \left(\phi_{0}\right) \dot{\phi}_{0} \sin \left(\theta_{0}\right)+\rho_{1} L_{1}^{2} \sin \left(\phi_{1}\right) \sin \left(\theta_{1}\right) \dot{L}_{0} \cos \left(\phi_{0}\right) \cos \left(\theta_{0}\right) \dot{\theta}_{0} \\
& -\rho_{1} L_{1}^{2} \sin \left(\phi_{1}\right) \cos \left(\theta_{1}\right) \dot{L}_{0} \sin \left(\phi_{0}\right) \dot{\phi}_{0} \cos \left(\theta_{0}\right)-\rho_{1} \dot{\phi}_{1} L_{1}^{2} \dot{L}_{1}-2 m_{1} L_{1} \dot{\phi}_{1} \dot{L}_{1}+m_{1} L_{1} \sin \left(\phi_{1}\right) \cos \left(\theta_{1}\right)
\end{aligned}
$$




$$
\begin{aligned}
& b_{6}=-m_{1} \cos \left(\phi_{1}\right) \cos \left(\theta_{1}\right)-\rho_{1} \ddot{L}_{1} L_{1}+1 / 2 \rho_{1} L_{1}^{2} \dot{\phi}_{1}^{2}+m_{1} L_{1} \dot{\phi}_{1}^{2}+1 / 2 \rho_{1} L_{0}^{2} \dot{\phi}_{0}^{2} \\
& -m_{1} \sin \left(\phi_{1}\right) \ddot{L}_{0} \sin \left(\phi_{0}\right)+1 / 2 \rho_{1} L_{0}^{2}\left(\cos \left(\phi_{0}\right)\right)^{2} \dot{\theta}_{0}^{2}+1 / 2 \rho_{1} L_{1}^{2}\left(\cos \left(\phi_{1}\right)\right)^{2} \dot{\theta}_{1}^{2} \\
& +m_{1} L_{1}\left(\cos \left(\phi_{1}\right)\right)^{2} \dot{\theta}_{1}^{2}+Q_{6}-m_{1} \ddot{L}_{1}+1 / 2 \rho_{1} \dot{L}_{0}^{2}-1 / 2 \rho_{1} \dot{L}_{1}^{2}-\rho_{1} g L_{0} \cos \left(\phi_{0}\right) \cos \left(\theta_{0}\right) \\
& -\rho_{1} \sin \left(\phi_{1}\right) \ddot{L}_{0} \sin \left(\phi_{0}\right) L_{1}-\rho_{1} g L_{1} \cos \left(\phi_{1}\right) \cos \left(\theta_{1}\right)-m_{1} \cos \left(\phi_{1}\right) \cos \left(\theta_{1}\right) \ddot{L}_{0} \cos \left(\phi_{0}\right) \cos \left(\theta_{0}\right) \\
& +m_{1} \cos \left(\phi_{1}\right) \sin \left(\theta_{1}\right) L_{0} \cos \left(\phi_{0}\right) \dot{\phi}_{0}^{2} \sin \left(\theta_{0}\right)+2 m_{1} \cos \left(\phi_{1}\right) \cos \left(\theta_{1}\right) \dot{L}_{0} \cos \left(\phi_{0}\right) \sin \left(\theta_{0}\right) \dot{\theta}_{0} \\
& +2 m_{1} \cos \left(\phi_{1}\right) \cos \left(\theta_{1}\right) \dot{L}_{0} \sin \left(\phi_{0}\right) \dot{\phi}_{0} \cos \left(\theta_{0}\right)+m_{1} \cos \left(\phi_{1}\right) \cos \left(\theta_{1}\right) L_{0} \cos \left(\phi_{0}\right) \dot{\phi}_{0}{ }^{2} \cos \left(\theta_{0}\right) \\
& -2 m_{1} \cos \left(\phi_{1}\right) \cos \left(\theta_{1}\right) L_{0} \sin \left(\phi_{0}\right) \dot{\phi}_{0} \sin \left(\theta_{0}\right) \dot{\theta}_{0}+m_{1} \cos \left(\phi_{1}\right) \cos \left(\theta_{1}\right) L_{0} \cos \left(\phi_{0}\right) \cos \left(\theta_{0}\right) \dot{\theta}_{0}^{2} \\
& +2 m_{1} \cos \left(\phi_{1}\right) \sin \left(\theta_{1}\right) \dot{L}_{0} \sin \left(\phi_{0}\right) \dot{\phi}_{0} \sin \left(\theta_{0}\right)+m_{1} \sin \left(\phi_{1}\right) L_{0} \sin \left(\phi_{0}\right) \dot{\phi}_{0}^{2} \\
& -2 m_{1} \sin \left(\phi_{1}\right) \dot{L}_{0} \cos \left(\phi_{0}\right) \dot{\phi}_{0}-\rho_{1} \cos \left(\phi_{1}\right) \cos \left(\theta_{1}\right) \ddot{L}_{0} \cos \left(\phi_{0}\right) \cos \left(\theta_{0}\right) L_{1} \\
& -\rho_{1} \cos \left(\phi_{1}\right) \sin \left(\theta_{1}\right) \ddot{L}_{0} \cos \left(\phi_{0}\right) \sin \left(\theta_{0}\right) L_{1}+2 \rho_{1} \cos \left(\phi_{1}\right) \sin \left(\theta_{1}\right) L_{0} \sin \left(\phi_{0}\right) \dot{\phi}_{0} \cos \left(\theta_{0}\right) \dot{\theta}_{0} L_{1} \\
& +\rho_{1} \cos \left(\phi_{1}\right) \sin \left(\theta_{1}\right) L_{0} \cos \left(\phi_{0}\right) \dot{\phi}_{0}^{2} \sin \left(\theta_{0}\right) L_{1}+2 \rho_{1} \cos \left(\phi_{1}\right) \cos \left(\theta_{1}\right) \dot{L}_{0} \sin \left(\phi_{0}\right) \dot{\phi}_{0} \cos \left(\theta_{0}\right) L_{1} \\
& +\rho_{1} \cos \left(\phi_{1}\right) \sin \left(\theta_{1}\right) L_{0} \cos \left(\phi_{0}\right) \sin \left(\theta_{0}\right) \dot{\theta}_{0}^{2} L_{1}-2 m_{1} \cos \left(\phi_{1}\right) \sin \left(\theta_{1}\right) \dot{L}_{0} \cos \left(\phi_{0}\right) \cos \left(\theta_{0}\right) \dot{\theta}_{0} \\
& +2 m_{1} \cos \left(\phi_{1}\right) \sin \left(\theta_{1}\right) L_{0} \sin \left(\phi_{0}\right) \dot{\phi}_{0} \cos \left(\theta_{0}\right) \dot{\theta}_{0}+m_{1} \cos \left(\phi_{1}\right) \sin \left(\theta_{1}\right) L_{0} \cos \left(\phi_{0}\right) \sin \left(\theta_{0}\right) \dot{\theta}_{0}^{2} \\
& -m_{1} \cos \left(\phi_{1}\right) \sin \left(\theta_{1}\right) \ddot{L}_{0} \cos \left(\phi_{0}\right) \sin \left(\theta_{0}\right)-2 \rho_{1} \cos \left(\phi_{1}\right) \sin \left(\theta_{1}\right) \dot{L}_{0} \cos \left(\phi_{0}\right) \cos \left(\theta_{0}\right) \dot{\theta}_{0} L_{1} \\
& +\rho_{1} \sin \left(\phi_{1}\right) L_{0} \sin \left(\phi_{0}\right) \dot{\phi}_{0}^{2} L_{1}+2 \rho_{1} \cos \left(\phi_{1}\right) \cos \left(\theta_{1}\right) \dot{L}_{0} \cos \left(\phi_{0}\right) \sin \left(\theta_{0}\right) \dot{\theta}_{0} L_{1} \\
& +\rho_{1} \cos \left(\phi_{1}\right) \cos \left(\theta_{1}\right) L_{0} \cos \left(\phi_{0}\right) \cos \left(\theta_{0}\right) \dot{\theta}_{0}^{2} L_{1}+\rho_{1} \cos \left(\phi_{1}\right) \cos \left(\theta_{1}\right) L_{0} \cos \left(\phi_{0}\right) \dot{\phi}_{0}^{2} \cos \left(\theta_{0}\right) L_{1} \\
& -2 \rho_{1} \cos \left(\phi_{1}\right) \cos \left(\theta_{1}\right) L_{0} \sin \left(\phi_{0}\right) \dot{\phi}_{0} \sin \left(\theta_{0}\right) \dot{\theta}_{0} L_{1}+2 \rho_{1} \cos \left(\phi_{1}\right) \sin \left(\theta_{1}\right) \dot{L}_{0} \sin \left(\phi_{0}\right) \dot{\phi}_{0} \sin \left(\theta_{0}\right) L_{1} \\
& -2 \rho_{1} \sin \left(\phi_{1}\right) \dot{L}_{0} \cos \left(\phi_{0}\right) \dot{\phi}_{0} L_{1}
\end{aligned}
$$

$$
\begin{aligned}
& b_{7}=-m_{2} L_{2} \cos \left(\phi_{2}\right) \sin \left(\theta_{2}\right) L_{0} \cos \left(\phi_{0}\right) \cos \left(\theta_{0}\right) \dot{\theta}_{0}^{2}+m_{2} L_{2} \cos \left(\phi_{2}\right) \sin \left(\theta_{2}\right)+Q_{7} \\
& -\rho_{2} L_{2}{ }^{2} \cos \left(\phi_{2}\right) \sin \left(\theta_{2}\right) \dot{L}_{0} \cos \left(\phi_{0}\right) \sin \left(\theta_{0}\right) \dot{\theta}_{0}+1 / 2 \\
& \rho_{2} L_{2}^{2} \cos \left(\phi_{2}\right) \cos \left(\theta_{2}\right) L_{0} \cos \left(\phi_{0}\right) \sin \left(\theta_{0}\right) \dot{\theta}_{0}^{2}-\rho_{2}\left(\cos \left(\phi_{2}\right)\right)^{2} \dot{\theta}_{2} L_{2}^{2} \dot{L}_{2} \\
& -1 / 2 \rho_{2} L_{2}{ }^{2} \cos \left(\phi_{2}\right) \sin \left(\theta_{2}\right) L_{0} \cos \left(\phi_{0}\right) \cos \left(\theta_{0}\right) \dot{\theta}_{0}^{2}-\rho_{2} L_{2}{ }^{2} \cos \left(\phi_{2}\right) \cos \left(\theta_{2}\right) \dot{L}_{0} \cos \left(\phi_{0}\right) \cos \left(\theta_{0}\right) \dot{\theta}_{0} \\
& +\rho_{2} L_{2}^{2} \cos \left(\phi_{2}\right) \cos \left(\theta_{2}\right) \dot{L}_{0} \sin \left(\phi_{0}\right) \dot{\phi}_{0} \sin \left(\theta_{0}\right)+1 / 2 \\
& \rho_{2} L_{2}^{2} \cos \left(\phi_{2}\right) \cos \left(\theta_{2}\right) L_{0} \cos \left(\phi_{0}\right) \dot{\phi}_{0}^{2} \sin \left(\theta_{0}\right)-1 / 2 \rho_{2} L_{2}^{2} \cos \left(\phi_{2}\right) \sin \left(\theta_{2}\right) L_{0} \cos \left(\phi_{0}\right) \dot{\phi}_{0}^{2} \cos \left(\theta_{0}\right) \\
& -2 m_{2} L_{2}\left(\cos \left(\phi_{2}\right)\right)^{2} \dot{\theta}_{2} \dot{L}_{2}-\rho_{2} L_{2}^{2} \cos \left(\phi_{2}\right) \sin \left(\theta_{2}\right) \dot{L}_{0} \sin \left(\phi_{0}\right) \dot{\phi}_{0} \cos \left(\theta_{0}\right)+1 / 2 \\
& \rho_{2} L_{2}{ }^{2} \cos \left(\phi_{2}\right) \sin \left(\theta_{2}\right) \ddot{L}_{0} \cos \left(\phi_{0}\right) \cos \left(\theta_{0}\right)+m_{2} L_{2} \cos \left(\phi_{2}\right) \cos \left(\theta_{2}\right) L_{0} \cos \left(\phi_{0}\right) \sin \left(\theta_{0}\right) \dot{\theta}_{0}^{2} \\
& +m_{2} L_{2} \cos \left(\phi_{2}\right) \sin \left(\theta_{2}\right) \ddot{L}_{0} \cos \left(\phi_{0}\right) \cos \left(\theta_{0}\right)-2 m_{2} L_{2} \cos \left(\phi_{2}\right) \sin \left(\theta_{2}\right) \dot{L}_{0} \sin \left(\phi_{0}\right) \dot{\phi}_{0} \cos \left(\theta_{0}\right) \\
& -2 m_{2} L_{2} \cos \left(\phi_{2}\right) \sin \left(\theta_{2}\right) \dot{L}_{0} \cos \left(\phi_{0}\right) \sin \left(\theta_{0}\right) \dot{\theta}_{0}+\rho_{2} L_{2}^{2} \cos \left(\phi_{2}\right) \sin \left(\theta_{2}\right) L_{0} \sin \left(\phi_{0}\right) \dot{\phi}_{0} \sin \left(\theta_{0}\right) \dot{\theta}_{0} \\
& +\rho_{2} L_{2}^{2} \cos \left(\phi_{2}\right) \cos \left(\theta_{2}\right) L_{0} \sin \left(\phi_{0}\right) \dot{\phi}_{0} \cos \left(\theta_{0}\right) \dot{\theta}_{0}-2 \\
& m_{2} L_{2} \cos \left(\phi_{2}\right) \cos \left(\theta_{2}\right) \dot{L}_{0} \cos \left(\phi_{0}\right) \cos \left(\theta_{0}\right) \dot{\theta}_{0}+1 / 2 \rho_{2} g \cos \left(\phi_{2}\right) \sin \left(\theta_{2}\right) L_{2}{ }^{2}+2 m_{2} L_{2}{ }^{2} \cos \left(\phi_{2}\right) \dot{\theta}_{2} \sin \left(\phi_{2}\right) \dot{\phi}_{2} \\
& +2 / 3 \rho_{2} \cos \left(\phi_{2}\right) \dot{\theta}_{2} L_{2}^{3} \sin \left(\phi_{2}\right) \dot{\phi}_{2}-1 / 2 \rho_{2} L_{2}^{2} \cos \left(\phi_{2}\right) \cos \left(\theta_{2}\right) \ddot{L}_{0} \cos \left(\phi_{0}\right) \sin \left(\theta_{0}\right) \\
& +2 m_{2} L_{2} \cos \left(\phi_{2}\right) \sin \left(\theta_{2}\right) L_{0} \sin \left(\phi_{0}\right) \dot{\phi}_{0} \sin \left(\theta_{0}\right) \dot{\theta}_{0}-m_{2} L_{2} \cos \left(\phi_{2}\right) \sin \left(\theta_{2}\right) L_{0} \cos \left(\phi_{0}\right) \dot{\phi}_{0}^{2} \cos \left(\theta_{0}\right) \\
& -m_{2} L_{2} \cos \left(\phi_{2}\right) \cos \left(\theta_{2}\right) \ddot{L}_{0} \cos \left(\phi_{0}\right) \sin \left(\theta_{0}\right)+2 m_{2} L_{2} \cos \left(\phi_{2}\right) \cos \left(\theta_{2}\right) L_{0} \sin \left(\phi_{0}\right) \dot{\phi}_{0} \cos \left(\theta_{0}\right) \dot{\theta}_{0} \\
& +2 m_{2} L_{2} \cos \left(\phi_{2}\right) \cos \left(\theta_{2}\right) \dot{L}_{0} \sin \left(\phi_{0}\right) \dot{\phi}_{0} \sin \left(\theta_{0}\right)+m_{2} L_{2} \cos \left(\phi_{2}\right) \cos \left(\theta_{2}\right) L_{0} \cos \left(\phi_{0}\right) \dot{\phi}_{0}^{2} \sin \left(\theta_{0}\right)
\end{aligned}
$$




$$
\begin{aligned}
& b_{8}=-2 m_{2} L_{2} \dot{\phi}_{2} \dot{L}_{2}-2 m_{2} L_{2} \sin \left(\phi_{2}\right) \sin \left(\theta_{2}\right) L_{0} \sin \left(\phi_{0}\right) \dot{\phi}_{0} \cos \left(\theta_{0}\right) \dot{\theta}_{0} \\
& -2 m_{2} L_{2} \sin \left(\phi_{2}\right) \sin \left(\theta_{2}\right) \dot{L}_{0} \sin \left(\phi_{0}\right) \dot{\phi}_{0} \sin \left(\theta_{0}\right)-m_{2} L_{2}^{2} \cos \left(\phi_{2}\right) \dot{\theta}_{2}^{2} \sin \left(\phi_{2}\right) \\
& -2 m_{2} L_{2} \cos \left(\phi_{2}\right) \dot{L}_{0} \cos \left(\phi_{0}\right) \dot{\phi}_{0}-m_{2} L_{2} \sin \left(\phi_{2}\right) \sin \left(\theta_{2}\right) L_{0} \cos \left(\phi_{0}\right) \dot{\phi}_{0}^{2} \sin \left(\theta_{0}\right) \\
& -m_{2} L_{2} \cos \left(\phi_{2}\right) \ddot{L}_{0} \sin \left(\phi_{0}\right)+1 / 2 \rho_{2} g \sin \left(\phi_{2}\right) \cos \left(\theta_{2}\right) L_{2}^{2}+2 m_{2} L_{2} \sin \left(\phi_{2}\right) \sin \left(\theta_{2}\right) \dot{L}_{0} \cos \left(\phi_{0}\right) \cos \left(\theta_{0}\right) \dot{\theta}_{0} \\
& +m_{2} L_{2} \cos \left(\phi_{2}\right) L_{0} \sin \left(\phi_{0}\right) \dot{\phi}_{0}{ }^{2}-1 / 2 \rho_{2} L_{2}{ }^{2} \cos \left(\phi_{2}\right) \ddot{L}_{0} \sin \left(\phi_{0}\right)-m_{2} L_{2} \sin \left(\phi_{2}\right) \sin \left(\theta_{2}\right) L_{0} \cos \left(\phi_{0}\right) \sin \left(\theta_{0}\right) \dot{\theta}_{0}{ }^{2} \\
& -m_{2} L_{2} \sin \left(\phi_{2}\right) \cos \left(\theta_{2}\right) L_{0} \cos \left(\phi_{0}\right) \dot{\phi}_{0}^{2} \cos \left(\theta_{0}\right)+m_{2} L_{2} \sin \left(\phi_{2}\right) \cos \left(\theta_{2}\right) \ddot{L}_{0} \cos \left(\phi_{0}\right) \cos \left(\theta_{0}\right) \\
& +2 m_{2} L_{2} \sin \left(\phi_{2}\right) \cos \left(\theta_{2}\right) L_{0} \sin \left(\phi_{0}\right) \dot{\phi}_{0} \sin \left(\theta_{0}\right) \dot{\theta}_{0}+1 / 2 \rho_{2} L_{2}^{2} \sin \left(\phi_{2}\right) \cos \left(\theta_{2}\right) \ddot{L}_{0} \cos \left(\phi_{0}\right) \cos \left(\theta_{0}\right) \\
& -m_{2} L_{2} \sin \left(\phi_{2}\right) \cos \left(\theta_{2}\right) L_{0} \cos \left(\phi_{0}\right) \cos \left(\theta_{0}\right) \dot{\theta}_{0}^{2}+\rho_{2} L_{2}^{2} \sin \left(\phi_{2}\right) \cos \left(\theta_{2}\right) L_{0} \sin \left(\phi_{0}\right) \dot{\phi}_{0} \sin \left(\theta_{0}\right) \dot{\theta}_{0} \\
& +1 / 2 \rho_{2} L_{2}^{2} \sin \left(\phi_{2}\right) \sin \left(\theta_{2}\right) \ddot{L}_{0} \cos \left(\phi_{0}\right) \sin \left(\theta_{0}\right)-1 / 2 \rho_{2} L_{2}^{2} \sin \left(\phi_{2}\right) \cos \left(\theta_{2}\right) L_{0} \cos \left(\phi_{0}\right) \cos \left(\theta_{0}\right) \dot{\theta}_{0}^{2} \\
& -1 / 2 \rho_{2} L_{2}^{2} \sin \left(\phi_{2}\right) \sin \left(\theta_{2}\right) L_{0} \cos \left(\phi_{0}\right) \dot{\phi}_{0}^{2} \sin \left(\theta_{0}\right)-1 / 3 \rho_{2} \cos \left(\phi_{2}\right) \dot{\theta}_{2}^{2} \sin \left(\phi_{2}\right) L_{2}^{3} \\
& -1 / 2 \rho_{2} L_{2}^{2} \sin \left(\phi_{2}\right) \cos \left(\theta_{2}\right) L_{0} \cos \left(\phi_{0}\right) \dot{\phi}_{0}^{2} \cos \left(\theta_{0}\right)+1 / 2 \rho_{2} L_{2}^{2} \cos \left(\phi_{2}\right) L_{0} \sin \left(\phi_{0}\right) \dot{\phi}_{0}^{2} \\
& -\rho_{2} L_{2}^{2} \sin \left(\phi_{2}\right) \cos \left(\theta_{2}\right) \dot{L}_{0} \cos \left(\phi_{0}\right) \sin \left(\theta_{0}\right) \dot{\theta}_{0}-\rho_{2} L_{2}^{2} \sin \left(\phi_{2}\right) \cos \left(\theta_{2}\right) \dot{L}_{0} \sin \left(\phi_{0}\right) \dot{\phi}_{0} \cos \left(\theta_{0}\right) \\
& +m_{2} L_{2} \sin \left(\phi_{2}\right) \sin \left(\theta_{2}\right) \ddot{L}_{0} \cos \left(\phi_{0}\right) \sin \left(\theta_{0}\right)-\rho_{2} \dot{\phi}_{2} L_{2}^{2} \dot{L}_{2}+m_{2} L_{2} \sin \left(\phi_{2}\right) \cos \left(\theta_{2}\right) \\
& -1 / 2 \rho_{2} L_{2}{ }^{2} \sin \left(\phi_{2}\right) \sin \left(\theta_{2}\right) L_{0} \cos \left(\phi_{0}\right) \sin \left(\theta_{0}\right) \dot{\theta}_{0}{ }^{2}-\rho_{2} L_{2}{ }^{2} \sin \left(\phi_{2}\right) \sin \left(\theta_{2}\right) L_{0} \sin \left(\phi_{0}\right) \dot{\phi}_{0} \cos \left(\theta_{0}\right) \dot{\theta}_{0} \\
& -\rho_{2} L_{2}^{2} \cos \left(\phi_{2}\right) \dot{L}_{0} \cos \left(\phi_{0}\right) \dot{\phi}_{0}-\rho_{2} L_{2}^{2} \sin \left(\phi_{2}\right) \sin \left(\theta_{2}\right) \dot{L}_{0} \sin \left(\phi_{0}\right) \dot{\phi}_{0} \sin \left(\theta_{0}\right) \\
& +\rho_{2} L_{2}^{2} \sin \left(\phi_{2}\right) \sin \left(\theta_{2}\right) \dot{L}_{0} \cos \left(\phi_{0}\right) \cos \left(\theta_{0}\right) \dot{\theta}_{0}-2 m_{2} L_{2} \sin \left(\phi_{2}\right) \cos \left(\theta_{2}\right) \dot{L}_{0} \sin \left(\phi_{0}\right) \dot{\phi}_{0} \cos \left(\theta_{0}\right) \\
& -2 m_{2} L_{2} \sin \left(\phi_{2}\right) \cos \left(\theta_{2}\right) \dot{L}_{0} \cos \left(\phi_{0}\right) \sin \left(\theta_{0}\right) \dot{\theta}_{0}+Q_{8} \\
& b_{9}=-\rho_{2} \ddot{L}_{2} L_{2}+1 / 2 \rho_{2} L_{2}^{2} \dot{\phi}_{2}^{2}+1 / 2 \rho_{2} L_{0}^{2} \dot{\phi}_{0}^{2}-m_{2} \cos \left(\phi_{2}\right) \cos \left(\theta_{2}\right) \\
& +m_{2} L_{2} \dot{\phi}_{2}^{2}-\rho_{2} \cos \left(\phi_{2}\right) \cos \left(\theta_{2}\right) \ddot{L}_{0} \cos \left(\phi_{0}\right) \cos \left(\theta_{0}\right) L_{2}+m_{2} \cos \left(\phi_{2}\right) \cos \left(\theta_{2}\right) L_{0} \cos \left(\phi_{0}\right) \cos \left(\theta_{0}\right) \dot{\theta}_{0}^{2} \\
& +m_{2} \cos \left(\phi_{2}\right) \cos \left(\theta_{2}\right) L_{0} \cos \left(\phi_{0}\right) \dot{\phi}_{0}^{2} \cos \left(\theta_{0}\right)+2 m_{2} \cos \left(\phi_{2}\right) \cos \left(\theta_{2}\right) \dot{L}_{0} \cos \left(\phi_{0}\right) \sin \left(\theta_{0}\right) \dot{\theta}_{0} \\
& +2 m_{2} \cos \left(\phi_{2}\right) \cos \left(\theta_{2}\right) \dot{L}_{0} \sin \left(\phi_{0}\right) \dot{\phi}_{0} \cos \left(\theta_{0}\right)+m_{2} \cos \left(\phi_{2}\right) \sin \left(\theta_{2}\right) L_{0} \cos \left(\phi_{0}\right) \sin \left(\theta_{0}\right) \dot{\theta}_{0}^{2} \\
& -2 m_{2} \cos \left(\phi_{2}\right) \sin \left(\theta_{2}\right) \dot{L}_{0} \cos \left(\phi_{0}\right) \cos \left(\theta_{0}\right) \dot{\theta}_{0}+m_{2} \cos \left(\phi_{2}\right) \sin \left(\theta_{2}\right) L_{0} \cos \left(\phi_{0}\right) \dot{\phi}_{0}^{2} \sin \left(\theta_{0}\right) \\
& +2 m_{2} \cos \left(\phi_{2}\right) \sin \left(\theta_{2}\right) \dot{L}_{0} \sin \left(\phi_{0}\right) \dot{\phi}_{0} \sin \left(\theta_{0}\right)-\rho_{2} \cos \left(\phi_{2}\right) \sin \left(\theta_{2}\right) \ddot{L}_{0} \cos \left(\phi_{0}\right) \sin \left(\theta_{0}\right) L_{2} \\
& -2 \rho_{2} \cos \left(\phi_{2}\right) \sin \left(\theta_{2}\right) \dot{L}_{0} \cos \left(\phi_{0}\right) \cos \left(\theta_{0}\right) \dot{\theta}_{0} L_{2}+2 \rho_{2} \cos \left(\phi_{2}\right) \sin \left(\theta_{2}\right) \dot{L}_{0} \sin \left(\phi_{0}\right) \dot{\phi}_{0} \sin \left(\theta_{0}\right) L_{2} \\
& +\rho_{2} \sin \left(\phi_{2}\right) L_{0} \sin \left(\phi_{0}\right) \dot{\phi}_{0}^{2} L_{2}-2 \rho_{2} \sin \left(\phi_{2}\right) \dot{L}_{0} \cos \left(\phi_{0}\right) \dot{\phi}_{0} L_{2} \\
& -2 m_{2} \cos \left(\phi_{2}\right) \cos \left(\theta_{2}\right) L_{0} \sin \left(\phi_{0}\right) \dot{\phi}_{0} \sin \left(\theta_{0}\right) \dot{\theta}_{0}-m_{2} \cos \left(\phi_{2}\right) \cos \left(\theta_{2}\right) \ddot{L}_{0} \cos \left(\phi_{0}\right) \cos \left(\theta_{0}\right) \\
& +2 m_{2} \cos \left(\phi_{2}\right) \sin \left(\theta_{2}\right) L_{0} \sin \left(\phi_{0}\right) \dot{\phi}_{0} \cos \left(\theta_{0}\right) \dot{\theta}_{0}-m_{2} \cos \left(\phi_{2}\right) \sin \left(\theta_{2}\right) \ddot{L}_{0} \cos \left(\phi_{0}\right) \sin \left(\theta_{0}\right) \\
& +\rho_{2} \cos \left(\phi_{2}\right) \cos \left(\theta_{2}\right) L_{0} \cos \left(\phi_{0}\right) \dot{\phi}_{0}{ }^{2} \cos \left(\theta_{0}\right) L_{2}+2 \rho_{2} \cos \left(\phi_{2}\right) \cos \left(\theta_{2}\right) \dot{L}_{0} \cos \left(\phi_{0}\right) \sin \left(\theta_{0}\right) \dot{\theta}_{0} L_{2} \\
& +2 \rho_{2} \cos \left(\phi_{2}\right) \cos \left(\theta_{2}\right) \dot{L}_{0} \sin \left(\phi_{0}\right) \dot{\phi}_{0} \cos \left(\theta_{0}\right) L_{2}+\rho_{2} \cos \left(\phi_{2}\right) \sin \left(\theta_{2}\right) L_{0} \cos \left(\phi_{0}\right) \dot{\phi}_{0}^{2} \sin \left(\theta_{0}\right) L_{2} \\
& +\rho_{2} \cos \left(\phi_{2}\right) \cos \left(\theta_{2}\right) L_{0} \cos \left(\phi_{0}\right) \cos \left(\theta_{0}\right) \dot{\theta}_{0}^{2} L_{2}+\rho_{2} \cos \left(\phi_{2}\right) \sin \left(\theta_{2}\right) L_{0} \cos \left(\phi_{0}\right) \sin \left(\theta_{0}\right) \dot{\theta}_{0}^{2} L_{2} \\
& -2 \rho_{2} \cos \left(\phi_{2}\right) \cos \left(\theta_{2}\right) L_{0} \sin \left(\phi_{0}\right) \dot{\phi}_{0} \sin \left(\theta_{0}\right) \dot{\theta}_{0} L_{2}+1 / 2 \rho_{2} L_{0}{ }^{2}\left(\cos \left(\phi_{0}\right)\right)^{2} \dot{\theta}_{0}{ }^{2}+m_{2} L_{2}\left(\cos \left(\phi_{2}\right)\right)^{2} \dot{\theta}_{2}^{2} \\
& +1 / 2 \rho_{2} L_{2}{ }^{2}\left(\cos \left(\phi_{2}\right)\right)^{2} \dot{\theta}_{2}^{2}+2 \rho_{2} \cos \left(\phi_{2}\right) \sin \left(\theta_{2}\right) L_{0} \sin \left(\phi_{0}\right) \dot{\phi}_{0} \cos \left(\theta_{0}\right) \dot{\theta}_{0} L_{2}-m_{2} \sin \left(\phi_{2}\right) \ddot{L}_{0} \sin \left(\phi_{0}\right) \\
& -m_{2} \ddot{L}_{2}+Q_{9}-\rho_{2} g L_{0} \cos \left(\phi_{0}\right) \cos \left(\theta_{0}\right)-\rho_{2} g L_{2} \cos \left(\phi_{2}\right) \cos \left(\theta_{2}\right)-\rho_{2} \sin \left(\phi_{2}\right) \ddot{L}_{0} \sin \left(\phi_{0}\right) L_{2} \\
& -2 m_{2} \sin \left(\phi_{2}\right) \dot{L}_{0} \cos \left(\phi_{0}\right) \dot{\phi}_{0}+m_{2} \sin \left(\phi_{2}\right) L_{0} \sin \left(\phi_{0}\right) \dot{\phi}_{0}{ }^{2}-1 / 2 \rho_{2} \dot{L}_{2}{ }^{2}+1 / 2 \rho_{2} \dot{L}_{0}{ }^{2}
\end{aligned}
$$

The above equations of motion are implemented in MATLAB and solved as a function of time by expressing them in state-space form.

\section{External Forces}

\section{Aerodynamic Kite Forces}

The major external forces acting on the kite-tow system that are not modeled thus far in the equations of motion are the lift and drag forces from the kite, together with the drag forces on the tether. The kite is assumed to be controlled by manipulating its angle of attack and roll angle. Thus, in this study, its attitude dynamics are ignored. The lift and drag forces due to the kite are derived using a velocity coordinate system, as shown in 4. 


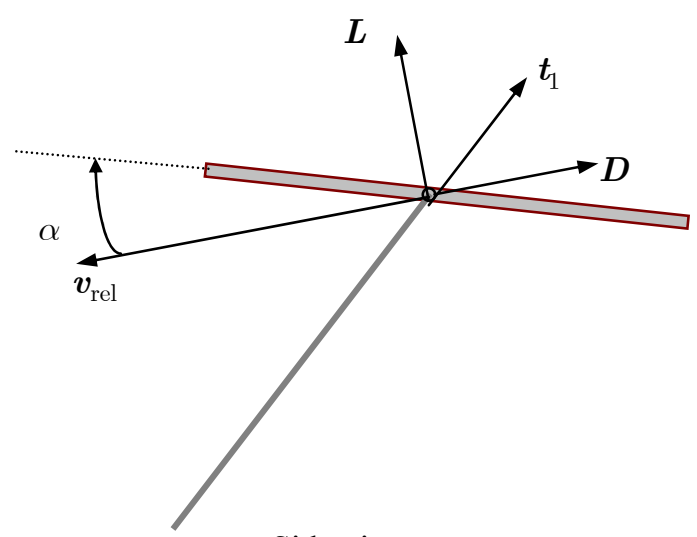

Side view

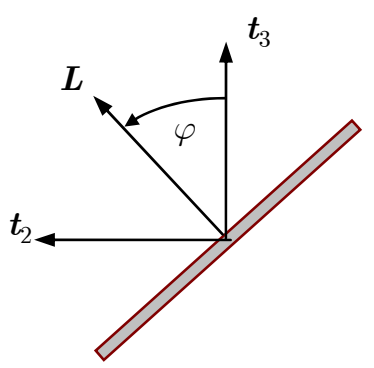

Back view

Fig. 4 Lift and drag forces on kite.

The vector that defines the plane containing the drag force and the velocity vector is given by

$$
\boldsymbol{t}_{2}=\frac{\boldsymbol{t}_{1} \times \boldsymbol{v}_{\text {rel }}}{\left|\boldsymbol{t}_{1} \times \boldsymbol{v}_{\text {rel }}\right|}
$$

where $t_{1}=\sin \theta_{j} \cos \phi_{j} i+\sin \phi_{j} j+\cos \theta_{j} \cos \phi_{j} k$ is a vector tangential to the $j$ th cable. The lift force, when the velocity roll angle is zero, is parallel to the vector

$$
t_{3}=\frac{\boldsymbol{v}_{\mathrm{rel}} \times \boldsymbol{t}_{2}}{\left|\boldsymbol{v}_{\mathrm{rel}} \times \boldsymbol{t}_{2}\right|}
$$

Hence, the lift and drag force vectors are defined according to

$$
\boldsymbol{L}=\frac{1}{2} \rho C_{L} S\left|\boldsymbol{v}_{\mathrm{rel}}\right|^{2}\left(\boldsymbol{t}_{2} \sin \varphi+\boldsymbol{t}_{3} \cos \varphi\right), \boldsymbol{D}=-\frac{1}{2} \rho C_{D} S\left|\boldsymbol{v}_{\mathrm{rel}}\right| \boldsymbol{v}_{\mathrm{rel}}
$$

Thus,

$$
F_{x}^{k}=(\boldsymbol{D}+\boldsymbol{L}) \cdot \boldsymbol{i}, \quad F_{y}^{k}=(\boldsymbol{D}+\boldsymbol{L}) \cdot \boldsymbol{j}, \quad F_{z}^{k}=(\boldsymbol{D}+\boldsymbol{L}) \cdot \boldsymbol{k}
$$

\section{Tether Drag Forces}

The drag forces on the tether are derived by assuming that the drag tangential to the tether is negligible. Therefore, the tether is discretized into a series of elements so that the contributions of drag from each element can be summed to obtain the total drag force and torque due to the tether. The magnitude of the relative wind component tangential to the cable is given by

$$
\boldsymbol{v}_{\mathrm{rel}} \cdot \boldsymbol{t}_{1}=\dot{L}-W_{x} \cos \phi \sin \theta-W_{y} \sin \phi-W_{z} \cos \phi \cos \theta
$$

The components of velocity normal to the cable are computed from

$$
\boldsymbol{v}_{n}=\boldsymbol{v}_{\text {rel }}-\left(\boldsymbol{v}_{\text {rel }} \cdot \boldsymbol{t}_{1}\right) \boldsymbol{t}_{1}
$$

The contribution of the tether drag to the generalized forces are obtained as

$$
\begin{gathered}
Q_{\theta}^{t}=-\sum_{j=1}^{n_{e}} \frac{1}{2} \rho_{j} C_{n} d \frac{L}{n_{e}} \boldsymbol{v}_{n_{j}}\left|\boldsymbol{v}_{n_{j}}\right| \cdot \frac{\partial R_{j}}{\partial \theta} \\
Q_{\phi}^{t}=-\sum_{j=1}^{n_{e}} \frac{1}{2} \rho_{j} C_{n} d \frac{L}{n_{e}} \boldsymbol{v}_{n_{j}}\left|\boldsymbol{v}_{n_{j}}\right| \cdot \frac{\partial R_{j}}{\partial \phi}
\end{gathered}
$$

The complete set of equations of motion may be integrated after first solving the coupled differential equations for the second derivatives of the generalized coordinates together with the normal reaction and tether tension. The resulting equations are cast in state-space form. 


\section{Numerical Optimization of Trajectories for the Kite Systems}

In this section, we implement the equations of motion in a numerical optimization package called DIRECT, which implements the Legendre pseudospectral method. ${ }^{13-14}$ This allows the kite motion to be optimized to minimize some specified cost function. In this paper, we simulate maximum power trajectories. For maximum power generation, the objective is to maximize the power generated by the system per cycle. The instantaneous power is given by $T \dot{L}$ (assuming a $100 \%$ efficient process). During one cycle, it is necessary to reel the tether out then back in again. This means that the tether tension must be judiciously controlled in such a way that the net energy per cycle is positive. In addition, because the kite model is highly simplified, it is desirable to maintain the kite control angles and reel acceleration as smooth as possible. Thus, we seek to minimize the following combined cost function

$$
J=\int_{t_{0}}^{t_{f}}\left[-\frac{T \dot{L}}{t_{f}-t_{0}}+W_{1} \sum_{n} u_{j}^{2}\right] \mathrm{d} t
$$

where $W_{1}$ is a weighting parameter, and $u_{j}$ is a control input into the system. The second term in the cost is used to prevent unrealistic jumps in the pseudocontrol inputs because we have not modeled low-level implementations of the controls via actuators. The kite control parameters are constrained by the following constraints

$$
|\alpha| \leq 10 \mathrm{deg},|\varphi| \leq 30 \mathrm{deg},|\dot{\alpha}| \leq 5 \mathrm{deg} / \mathrm{s},|\dot{\varphi}| \leq 5 \mathrm{deg} / \mathrm{s},
$$

In the case where the tether is allowed to vary in length, some additional constraints are necessary. Controlling the tether length through the reel acceleration potentially allows rapid changes in tether tension. Because the tether cannot sustain compressive forces, a path constraint of the form

$$
T \geq T_{\min }
$$

must be enforced. In this paper, $T_{\min }$ is set as $5 \mathrm{~N}$. The following additional constraints are imposed on the reeling

$$
\left|\frac{\Delta L}{L_{\text {ref }}}\right| \leq 0.3,|\dot{L}| \leq 20 \mathrm{~m} / \mathrm{s},|\ddot{L}| \leq 6 \mathrm{~m} / \mathrm{s}^{2}
$$

In general, the cycling period should be free to be optimized. However, we have fixed it here to be 20 sec.

\section{A. Numerical Results}

We consider a system with kites weighing $50 \mathrm{~kg}$ each, with effective areas of $250 \mathrm{~m}^{2}$ each. The nominal cable lengths are $2 \mathrm{~km}$ and the wind speed is $10 \mathrm{~m} / \mathrm{s}$. Fig. 5 shows results for power generation with two-kites on the same line, and Fig. 6 shows results for the case of power generation with a branched line. Unfortunately, the constraint on the reel acceleration was scaled to be half that for the single line case, so the results for the branched case are less optimal than for the single case. However, the results are qualitatively quite similar. The advantage of the branched line case is that the cable drag influence is smaller. The systems are generating about $2.5 \mathrm{MW}$, but this is strongly influenced by the cycling time, the angle of attack constraints, and the reeling constraints. The results are similar to previously generated optimal trajectories for kites 

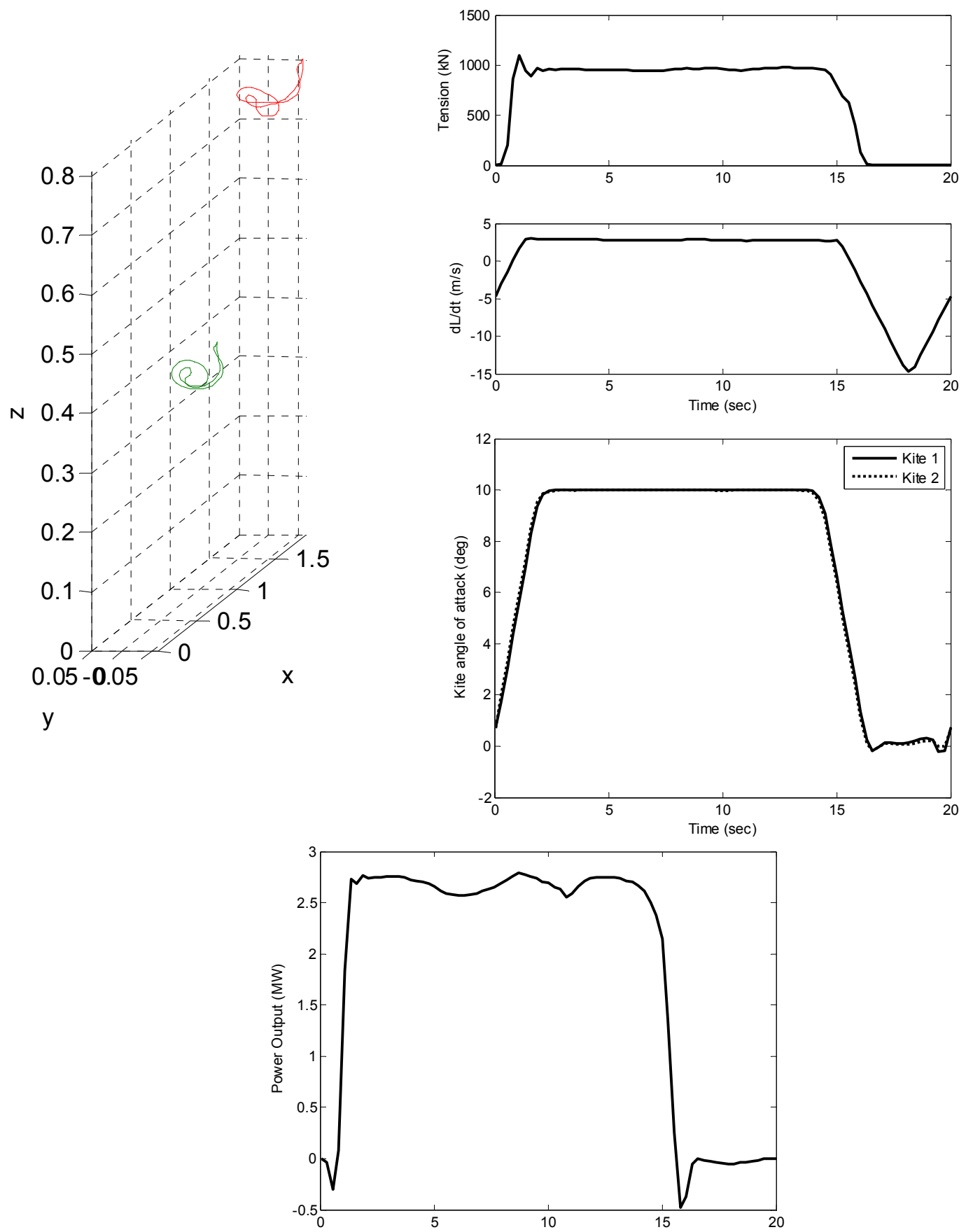

Fig. 5. Power generation using two-kites on single line. 

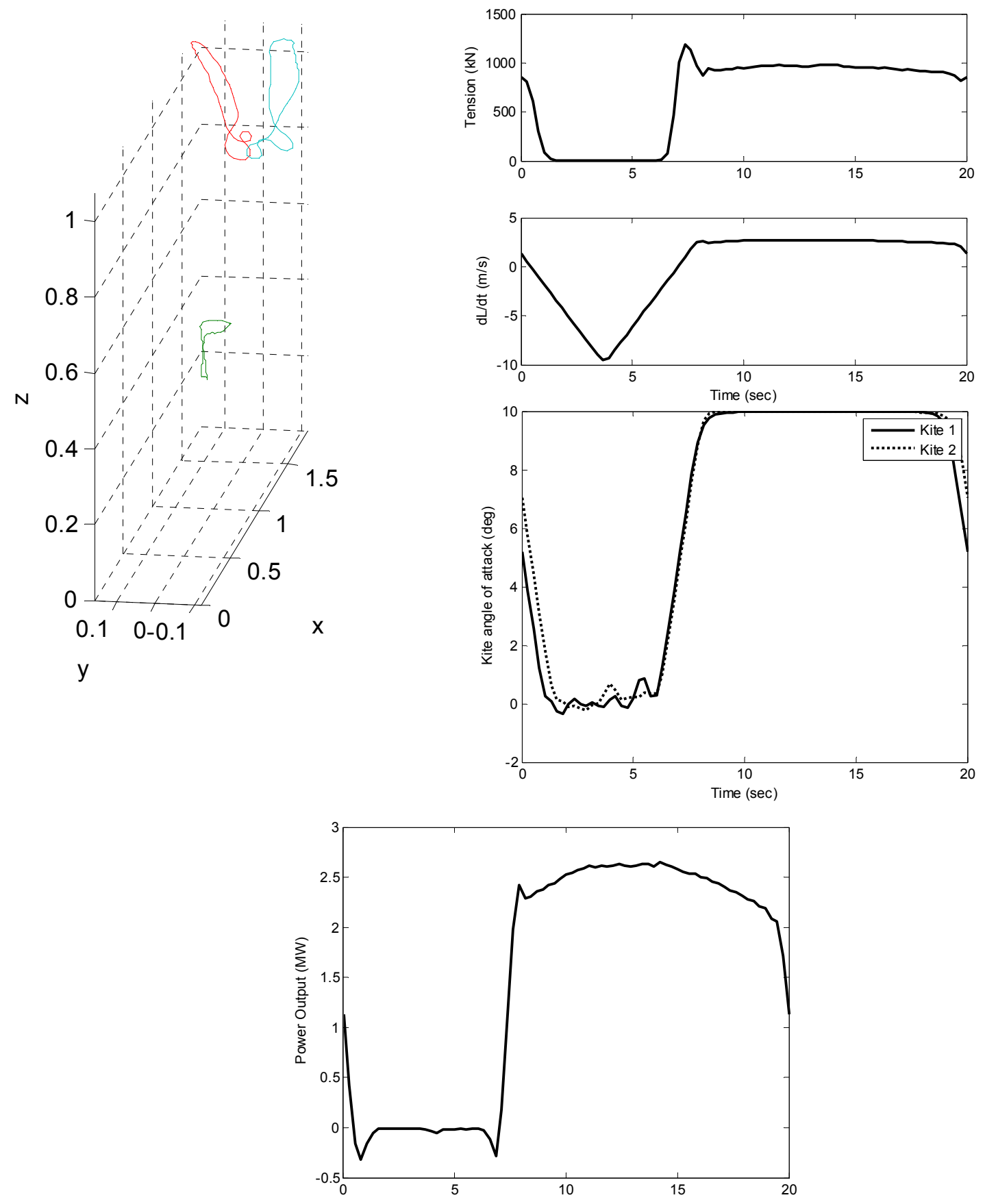

Fig. 6. Power generation with two-kites on branched line.

\section{Conclusions}

Mathematical modeling of multiple kite power generating systems enables predictions of system performance to be undertaken. For realistic results, numerical optimization needs to be performed to determine the power generating capacity for particular systems. Two different system configurations were simulated. The first considered multiple kites connected along the same line, whereas the second is the case of multiple kites connected via cable branching. The high frequency modes associated with longitudinal elasticity are removed from the analysis by treating the tethers as rigid. The constraint forces are determined at each time step as an output from the 
coupled equations of motion. Simulated results illustrate the generation of $2.5 \mathrm{MW}$ of power. The models will be useful for further research and analysis of kite systems.

\section{References}

${ }^{1}$ Carpenter, H.G., “Tethered Aircraft Having Remotely Controlled Angle of Attack," US Patent 5,931,416.

${ }^{2}$ Carpenter, H.G., "Tethered Aircraft System for Gathering Energy From Wind," US Patent 6,254,034.

3 Williams, P., Lansdorp, B., and Ockels, W., "Optimal Cross-Wind Towing and Power Generation with Tethered Kites," Journal of Guidance, Control, and Dynamics, Vol. 31, No. 1, 2008, pp.81-93.

${ }^{4}$ Ockels, W.J., "Laddermill, a Novel Concept to Exploit the Energy in the Airspace," Aircraft Design, Vol. 4, 2001, pp.8197.

${ }^{5}$ Lansdorp, B., and Ockels, W.J., "Comparison of Concepts for High-Altitude Wind Energy Generation with Ground Based Generator," Proceedings of the NRE 2005 Conference, Beijing, China, pp.409-417.

${ }^{6}$ Lansdorp, B., Remes, B., and Ockels, W.J., "Design and Testing of a Remotely Controlled Surfkite for the Laddermill," World Wind Energy Conference, Melbourne, Australia, Nov. 2005.

${ }^{7}$ Lansdorp, B., and Williams, P., "The Laddermill - Innovative Wind Energy from High Altitudes in Holland and Australia," Paper presented at Wind Power 2006, Adelaide, Australia, September 2006.

${ }^{8}$ Loyd, M.L., "Crosswind Kite Power," Journal of Energy, Vol. 4, No. 3, 1980, pp.106-111.

${ }^{9}$ Williams, P., Lansdorp, B., and Ockels, W., "Flexible Tethered Kite with Moveable Attachment Points, Part I: Dynamics and Control", AIAA Atmospheric Flight Mechanics Conference, Aug. 2007, AIAA Paper 2007-6628.

${ }^{10}$ Williams, P., Lansdorp, B., and Ockels, W., "Optimal Trajectories for Tethered Kite Mounted on a Vertical Axis Generator," AIAA Modeling and Simulation Conference, Aug. 2007, AIAA Paper 2007-6706.

${ }^{11}$ Lansdorp, B., "Towards Flight Testing of Remotely Controlled Surfkites for Wind Energy Generation," AIAA Paper 20076643, Aug. 2007.

${ }^{12}$ Houska, B., and Diehl, M., "Optimal Control of Power Generating Kites," European Control Conference 2007, CD-ROM.

${ }^{13}$ Elnagar, J., Kazemi, M.A., and Razzaghi, M., "The Pseudospectral Legendre Method for Discretizing Optimal Control Problems," IEEE Transactions on Automatic Control, Vol. 40, No. 10, 1995, pp.1793-1796.

${ }^{14}$ Ross, I.M., and Fahroo, F., "Legendre Pseudospectral Approximations of Optimal Control Problems," Lecture Notes in Control and Information Sciences, Vol. 295, Springer-Verlag, New York, pp.327-342. 